\title{
MVP: An Efficient CNN Accelerator with Matrix, Vector, and Processing-Near-Memory Units
}

\author{
SUNJUNG LEE, JAEWAN CHOI, WONKYUNG JUNG, BYEONGHO KIM, and JAEHYUN \\ PARK, Department of Intelligence and Information, Seoul National University, South Korea \\ HWEESOO KIM, Samsung Electronics Co., Ltd, South Korea \\ JUNG HO AHN, Department of Intelligence and Information \& Inter-University Semiconductor Research \\ Center, Seoul National University, South Korea
}

\begin{abstract}
Mobile and edge devices become common platforms for inferring convolutional neural networks (CNNs) due to superior privacy and service quality. To reduce the computational costs of convolution (CONV), recent CNN models adopt depth-wise CONV (DW-CONV) and Squeeze-and-Excitation (SE). However, existing area-efficient $\mathrm{CNN}$ accelerators are sub-optimal for these latest $\mathrm{CNN}$ models because they were mainly optimized for compute-intensive standard CONV layers with abundant data reuse that can be pipelined with activation and normalization operations. In contrast, DW-CONV and SE are memory-intensive with limited data reuse. The latter also strongly depends on the nearby CONV layers, making an effective pipelining a daunting task. Therefore, DW-CONV and SE only occupy $10 \%$ of entire operations but become memory bandwidth bound, spending more than $60 \%$ of the processing time in systolic-array-based accelerators.

We propose a CNN acceleration architecture called MVP, which efficiently processes both compute- and memory-intensive operations with a small area overhead on top of the baseline systolic-array-based architecture. We suggest a specialized vector unit tailored for processing DW-CONV, including multipliers, adder trees, and multi-banked buffers to meet the high memory bandwidth requirement. We augment the unified buffer with tiny processing elements to smoothly pipeline SE with the subsequent CONV, enabling concurrent processing of DW-CONV with standard CONV, thereby achieving the maximum utilization of arithmetic units. Our evaluation shows that MVP improves performance by $2.6 \times$ and reduces energy by $47 \%$ on average for EfficientNet-B0/B4/B7, MnasNet, and MobileNet-V1/V2 with only a 9\% area overhead compared to the baseline.
\end{abstract}

CCS Concepts: • Computer systems organization $\rightarrow$ Neural networks;

Additional Key Words and Phrases: Neural Networks, CNN, energy-efficient AI accelerator

\begin{abstract}
This work was supported by Samsung Advanced Institute of Technology and the Engineering Research Center Program through the National Research Foundation of Korea (NRF) funded by the Korean Government MSIT (NRF2018R1A5A1059921). The EDA tool was supported by the IC Design Education Center.

Authors' addresses: S. Lee, J. Choi, W. Jung, B. Kim, and J. Park, Department of Intelligence and Information, Seoul National University, 1 Gwanak-ro, Seoul, 08826, South Korea; emails: \{sjlee0407, jwchoi, jungwk, bhkim, jhpark\}@ scale.snu.ac.kr; H. Kim, Samsung Electronics Co., Ltd, 1-1 Samsungjeonja-ro, Hwaseong, Gyeonggi, 18448, South Korea; email: hs5006.kim@scale.snu.ac.kr; J. H. Ahn, Department of Intelligence and Information \& Research Institute for Convergence Science, Seoul National University, 1 Gwanak-ro, Seoul, 08826, South Korea; email: gajh@snu.ac.kr.
\end{abstract}

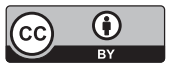

This work is licensed under a Creative Commons Attribution International 4.0 License.

(C) 2022 Copyright held by the owner/author(s).

1084-4309/2022/06-ART42

https://doi.org/10.1145/3497745

ACM Transactions on Design Automation of Electronic Systems, Vol. 27, No. 5, Article 42. Pub. date: June 2022. 
ACM Reference format:

Sunjung Lee, Jaewan Choi, Wonkyung Jung, Byeongho Kim, Jaehyun Park, Hweesoo Kim, and Jung Ho Ahn. 2022. MVP: An Efficient CNN Accelerator with Matrix, Vector, and Processing-Near-Memory Units. ACM Trans. Des. Autom. Electron. Syst. 27, 5, Article 42 (June 2022), 25 pages.

https://doi.org/10.1145/3497745

\section{INTRODUCTION}

Convolutional neural networks (CNNs) are used in a variety of applications such as image recognition and object detection. Mobile and edge devices are becoming a primary platform for CNN inference due to superior response time, security, and privacy. Major smartphone companies such as Apple, Google, and Samsung deploy CNN inference engines in their devices [3, 17, 46]. By 2022, 7.3 billion mobile device users are projected to have on-device artificial intelligence (AI) capability [15, 47], spurring the need for efficient CNN inference accelerators.

As standard convolution (ST-CONV) layers dominated the computation of conventional CNN models, a large body of accelerator research has focused on this layer type $[5-7,13,14,30,37$, $55,56,61]$. Among those, accelerators supporting systolic execution or its variants $[30,38]$ are gaining popularity due to superior performance, energy, and area efficiency. These architectures efficiently exploit massive parallelism and abundant data reuse opportunities of input feature maps (IFmaps) in computing output feature maps (OFmaps) for CONV layers. Hence, major industry players such as Google have already adopted this systolic-array-based acceleration in their production $[16,30]$.

The CNN models have evolved to reduce the arithmetic operations while retaining or improving recognition accuracy. In the early days, designers manually determined the model configurations, such as layer types, depth (the number of layers), and kernel sizes. ST-CONV with a kernel size of $3 \times 3$ or larger was dominant. As these ST-CONV layers are computationally heavy, various CONV types have been proposed to reduce the number of operations by changing the kernel shape or reducing the number of channels being referenced $[11,49,50]$.

The advent of neural architecture search (NAS [63]) has led to several changes in configuring CNNs. Early NAS studies reduce the number of operations drastically by mostly using STCONV with $1 \times 1$ kernel size (called point-wise CONV, PW-CONV) and depth-wise CONV (DW-CONV). However, due to excessive use of skip connection and unstructured layer configurations, the actual execution time was longer than the time predicted by the number of arithmetic operations. Therefore, the latest CNN models constructed by NAS utilize a building block (BB) composed of PW-CONV, DW-CONV, and squeeze-and-excitation (SE) as a unit since [51], in which memory-intensive operations become prevalent.

The systolic array (SysAr) architecture has structural limitations in handling these emerging, memory-intensive CNN operations. DW-CONV only uses a single IFmap to create a single OFmap. Therefore, SysAr that feeds a massive number of MACs in its matrix unit (MU) using a limited bandwidth from the unified buffer (UB) in a wavefront manner cannot fully utilize its MACs on DW-CONV. The other accelerator [8] fully utilizes the MACs it owns by arranging those with distributed register files and hierarchical on-chip networks, although at a significant cost in the area [33] and wire energy [18]. When normalization (NORM) and activation (ACT) operations are performed prior to CONV or SE depends on the nearby CONV layers, SysAr becomes inefficient as its MU and vector unit (VU) cannot be pipelined. PW-CONV occupies more than $90 \%$ of the number of operations but takes under $40 \%$ in execution time (see Figure 1). Memory-intensive operations such as DW-CONV and SE-Scale (the operation of applying the squeezed weights to feature maps) take the remaining $60 \%$ of execution time, demonstrating the importance of accelerating memory-intensive operations for the latest CNNs. 


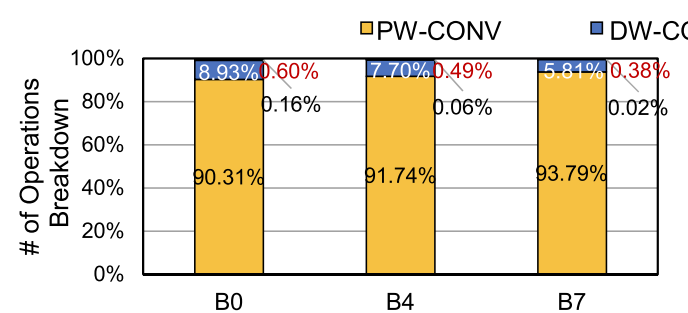

(a)

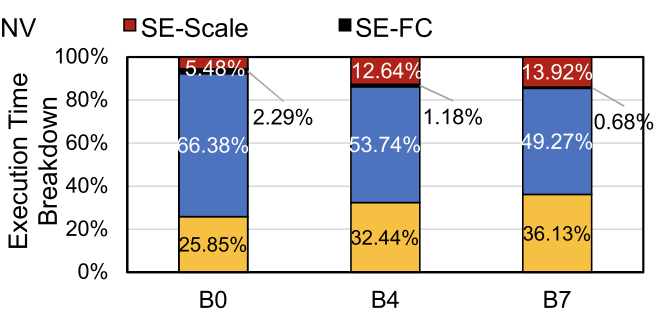

(b)

Fig. 1. (a) The number of operations and (b) execution time breakdown of EfficientNet-B0/B4/B7 of the evaluated systolic-array-based accelerator (configurations are specified in Section 4.1). NORM, ACT, and POOL operations are not included as their execution time is hidden through pipelining.

In this paper, we propose an MVP architecture composed of Matrix, Vector, and Processingnear-memory units (more accurately, processing-near-on-chip-unified-memory units) to efficiently process both compute- and memory-intensive CNN operations at a small area overhead on the baseline SysAr architecture. VU of SysAr is augmented to effectively process DW-CONV by adding an array of arithmetic units, whose size is much smaller than that of MU. VU stores a tile of an OFmap produced by PW-CONV in small-but-high-throughput buffers and performs DW-CONV with the array of multipliers and adder trees, exploiting the key characteristic of DWCONV that the reuse distance of its IFmaps is short. Processing-near-memory unit (PNMU) performs element-wise operations (NORM, ACT, and SE-Scale) that are processed immediately prior to CONV, which are performed at MU, to eliminate unnecessary on-/off-chip memory access. VU and PNMU significantly reduce the execution time of memory-intensive operations as these operations can mostly be overlapped with computation for the nearby PW-CONV layers.

This paper makes the following key contributions:

- We categorize the layer configurations of various CNN models and identify that the latest CNN models are composed of BBs that utilize PW-CONV, DW-CONV, and SE.

- We identify that the arithmetic resource of systolic-array-based CNN accelerators is severely underutilized when processing the memory-intensive operations prevalent in the BBs of the latest $\mathrm{CNN}$ models.

- We propose an MVP architecture that combines Matrix, Vector, and Processing-nearmemory units to efficiently process both compute- and memory-intensive operations of the latest CNN models with a small area overhead.

- MVP improves performance by $2.6 \times$ and reduces energy by $47 \%$ on average over EfficientNetB0/B4/B7, MnasNet, and MobileNet-V1/V2 with a $9 \%$ area overhead over the baseline SysAr.

\section{BACKGROUND AND MOTIVATION}

\subsection{Various Types of Convolution (CONV) Operations}

ST-CONV is a basic type of CONV where the size of a kernel, $K H \times K W$, is typically larger than $1 \times 1$. It convolutes IFmaps and weights to produce OFmaps, requiring $K H \times K W \times I C \times O H \times O W \times O C$ operations in total (see Figure 2(a)). Recent CNN models adopt new types of CONV layers to reduce the number of operations, either by limiting the number of input/output channels or using smaller kernels.

Grouped CONV (GR-CONV) and depth-wise CONV (DW-CONV) reduce the number of required operations compared to ST-CONV by dividing the input channels into multiple groups of channels. We call the number of groups $n G R$. GR-CONV requires $K H \times K W \times\left(\frac{I C}{n G R}\right) \times$ 


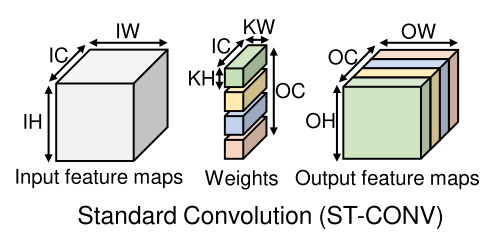

(a)

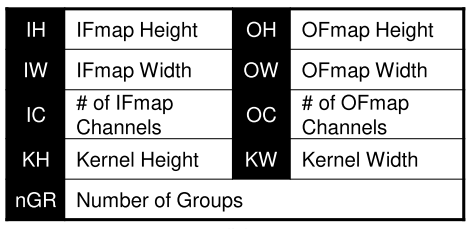

(b)

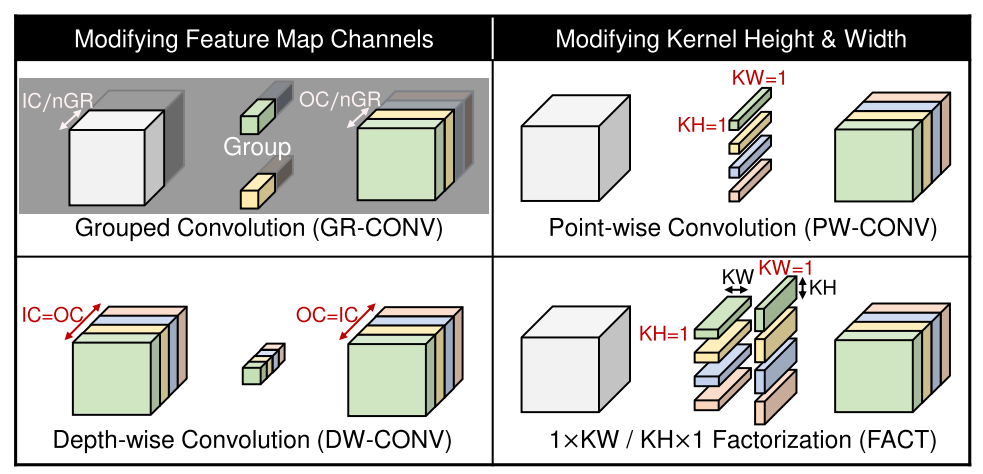

(c)

(d)

Fig. 2. (a) ST-CONV (in general, ST-CONV includes PW-CONV, but we distinguish the two terms to emphasize whether the kernel size is $1 \times 1$ or not), (b) abbreviations for IFmaps, OFmaps and weights, (c) CONVs with different ways to refer channels, and (d) CONVs with different kernel configurations.

$O H \times O W \times\left(\frac{O C}{n G R}\right) \times n G R$ operations, which are reduced by a factor of $n G R$ times compared to ST-CONV (see Figure 2(c)). DW-CONV is an extreme form of GR-CONV where $n G R=I C=O C$.

Point-wise CONV (PW-CONV) and Factorization (FACT) reduce the number of operations by using smaller kernels (see Figure $2(\mathrm{~d})$ ). PW-CONV exploits $1 \times 1$ kernels. FACT factorizes a $K H \times K W$ kernel into two: one with a $1 \times K H$ size and the other with a $K W \times 1$ size. Then it performs convolution for each of the two kernels, thereby reducing the total number of operations by a factor of $\frac{(K H \times K W)}{(K H+K W)}$ times.

\subsection{Trends in CNN Model Architecture}

Various CNN models have been proposed to increase the accuracy of image recognition and, at the same time, reduce the burden of computation (see Figure 3).

Handcrafted models: In the early days, many researchers tried empirical studies, tuning myriads of parameters such as layer types, number of layers, channel size, and resolution by hand to improve the accuracy of $\mathrm{CNN}$ models. As the number of layers increases, the accuracy tends to increase, albeit with the following issues. First, CNN models are computationally hungry. Early $\mathrm{CNN}$ models populated just a few layers, but later models use dozens of layers or more, requiring lots of arithmetic operations (e.g., VGGNet [45] requires about 20G operations). Therefore, GoogleNet [49], Inception-V3 [50], Xception [11], and ResNext [59] adopted PW-CONV, FACT, DW-CONV, and GR-CONV, respectively, to reduce the number of arithmetic operations. Second, there was a time when training was nearly impossible if the number of layers exceeds a certain threshold due to a vanishing gradient problem during backpropagation. ResNet [21] addressed this problem using skip connection, paving the way for stacking hundreds of layers. Batch normalization (BN) [27] and SE [25] were proposed to further increase the accuracy of CNN models by changing the entire Fmap elements using element-wise operations. 


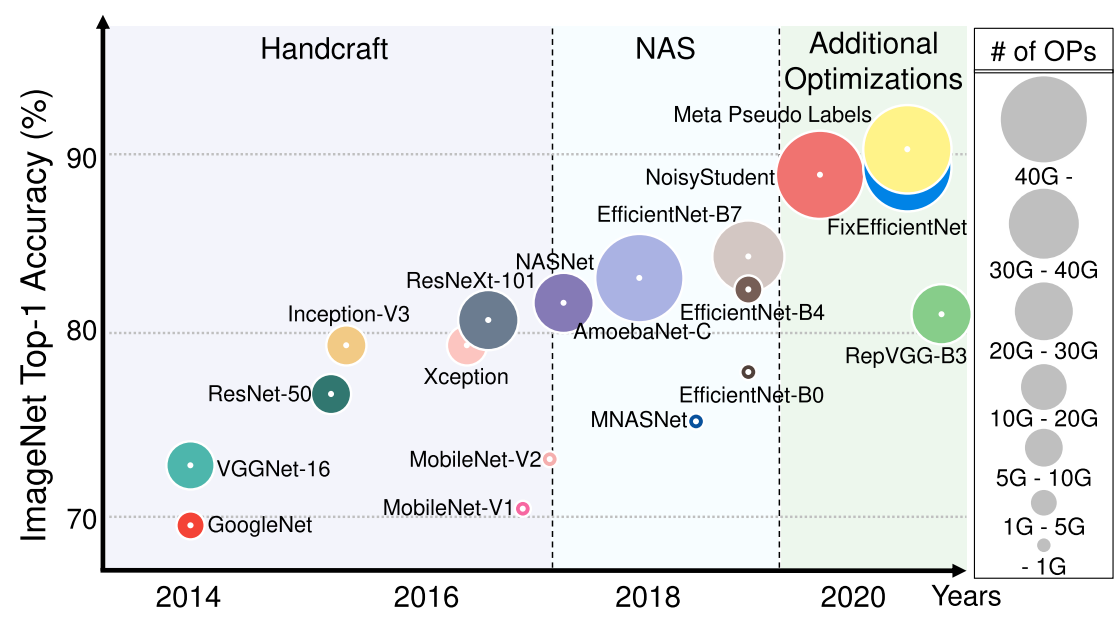

Fig. 3. Top-1 accuracy and the number of OPs of CNN models by year. Handcraft refers to the CNN models in which various configurations are manually tuned by researchers until mid-2017. The radius of a circle is proportional to the number of MAC operations required by the models.

NAS models: Because manually developing CNN models requires significant engineering efforts, NAS has been proposed to design the models automatically. NAS uses machine learning to explore efficient models in performance, computation complexity, and memory capacity by using various layer types developed in the handcrafted models as candidates. In the early NAS works, NASNet [63] and AmoebaNet [41] obtained high accuracy, but they required a huge amount of operations and suggested complex structures. Thus, the actual hardware execution time was much longer than the execution time predicted by the number of operations. To solve this problem, MnasNet [51] simplified the model structure using a BB, which was first used in ResNet, as a candidate for design search. EfficientNet [52] expanded MobileNet-V2 [44] and MnasNet to obtain high accuracy with fewer operations than the early NAS models.

Additional optimizations: Because EfficientNet demonstrated strength in computational complexity and accuracy to the other CNN models, subsequent studies tend to use it as a baseline model. NoisyStudent [58] increased the number of layers, channel size, and resolution of EfficientNet and changed the training method to perform pre-processing using ImageNet data and then proceed with self-training using Instagram data. FixEfficientNet [54] improved accuracy by applying image augmentation to both training and test time using EfficientNet. Meta Pseudo Labels [40] achieved a top-1 accuracy of over $90 \%$ for the first time using EfficientNet as a baseline model. Meta Pseudo Labels used knowledge distillation [23] which trains a student network with a pretrained teacher network. For the training, it used the ImageNet dataset as labeled data and the JFT-300M [23, 48] dataset as unlabeled data. RepVGG [12] is designed to achieve high utilization on conventional accelerators or GPUs. RepVGG uses skip connection for training and excludes it for inference to simplify the model and to reduce inference time. Also, RepVGG does not use DWCONV and PW-CONV which undergo low utilization; instead it only uses ST-CONV like VGGNet. Although RepVGG is suitable for conventional hardware, it has lower top-1 accuracy compared to the EfficientNet variants.

Even though the components of the $\mathrm{CNN}$ models have been changed over time, there is little change in the fundamental structure and the types of CONV in CNN models since MnasNet. CNN models mainly use fixed compositions consisting of repeated BBs, including PW-CONV, DW-CONV, and SE (Table 1). PW-CONV and DW-CONV are popular as they are the most efficient to reduce 
Table 1. Categorization of Operation Types Used in Various CNN Models

\begin{tabular}{cccccccc}
\hline & ST & PW & FACT & DW & GR & BB & SE \\
\hline VGGNet [45] & $\checkmark$ & & & & & & \\
GoogleNet [49] & $\checkmark$ & $\checkmark$ & & & & & \\
ResNet [21] & $\checkmark$ & $\checkmark$ & & & & $\checkmark$ & \\
Inception-V3 [50] & $\checkmark$ & $\checkmark$ & $\checkmark$ & & & & \\
Xception [11] & $* \checkmark$ & $\checkmark$ & & $\checkmark$ & & & \\
ResNeXt [59] & $* \checkmark$ & $\checkmark$ & & & $\checkmark$ & $\checkmark$ & \\
MobileNet-V1 [24] & $* \checkmark$ & $\checkmark$ & & $\checkmark$ & & & \\
MobileNet-V2 [44] & $* \checkmark$ & $\checkmark$ & & $\checkmark$ & & $\checkmark$ & \\
NASNet [63] & $* \checkmark$ & $\checkmark$ & & $\checkmark$ & & & \\
AmoebaNet [41] & $* \checkmark$ & $\checkmark$ & $\checkmark$ & $\checkmark$ & & & \\
MnasNet [51] & $* \checkmark$ & $\checkmark$ & & $\checkmark$ & & $\checkmark$ & $\checkmark$ \\
EfficientNet [52] & $* \checkmark$ & $\checkmark$ & & $\checkmark$ & & $\checkmark$ & $\checkmark$ \\
NoisyStudent [58] & $* \checkmark$ & $\checkmark$ & & $\checkmark$ & & $\checkmark$ & $\checkmark$ \\
FixEfficientNet [54] & $* \checkmark$ & $\checkmark$ & & $\checkmark$ & & $\checkmark$ & $\checkmark$ \\
Meta Pseudo Labels [40] & $* \checkmark$ & $\checkmark$ & & $\checkmark$ & & $\checkmark$ & $\checkmark$ \\
RepVGG [12] & $\checkmark$ & & & & & & \\
\hline
\end{tabular}

(* means that it is only used at the initial layer.)

the number of operations. BB is used to simplify the structure of the models. SE, like BN, increases accuracy by considering the degree of influence between Fmaps with a small computational cost. In contrast, ST-CONV is used only as the initial layer in the latest CNN models due to its high computational cost. GR-CONV does not appear in the latest CNN models, and FACT is rarely used even if they are candidates of NAS.

\subsection{EfficientNet: A State-of-the-art CNN Model}

EfficientNet [52] is a representative of more recent $\mathrm{CNN}$ models that use multiple BBs to achieve high accuracy with a relatively low computational cost. EfficientNet is an expanded structure of MobileNet-V2 and MnasNet that scales all three parameters (resolution, the number of channels, and the number of layers) concurrently as opposed to previous works [21, 24, 49] which scale only subsets of the three dimensions. EfficientNet introduces eight exemplar models (EfficientNetB0 B7), which require 4 20× lower computational costs compared to the previous CNN models with similar recognition accuracy. For example, both EfficientNet-B0 and ResNet-50 achieve nearly $77 \%$ Top- 1 accuracy, but they require $390 \mathrm{M}$ and $4.1 \mathrm{G}$ operations, respectively.

EfficientNet is a sequence of mobile inverted BBs (MBConvs). MBConv consists of the first PW-CONV followed by DW-CONV, SE, and the second PW-CONV (see Figure 4), where each CONV has trailing NORM and ACT layers. SE includes global average pooling (SE-AvgPOOL), fully connected layer (SE-FC), activation (SE-ACT), and element-wise multiplication (SE-Scale). SE first calculates scale factors for each channel using OFmap created by DW-CONV and then operates SE-Scale using the scale factors and the DW-CONV. There is a data dependency between DW-CONV and SE because each step uses the whole OFmaps directly. In step (1), SE-AvgPOOL needs all of the Fmap elements to compute the average, and SE-FC needs all of the channels to get scale factors per channel. In step (2), SE-Scale multiplies the scale factors with 


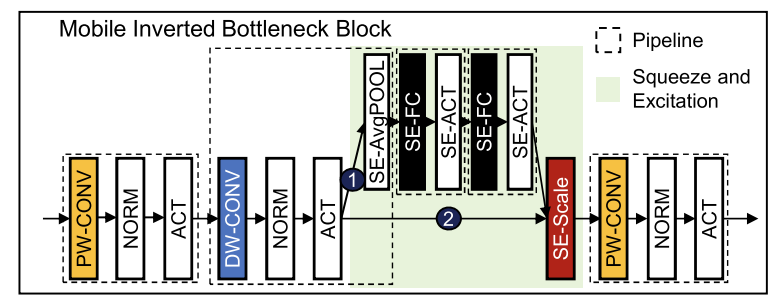

Fig. 4. Mobile inverted bottleneck block (MBConv) of EfficientNet.

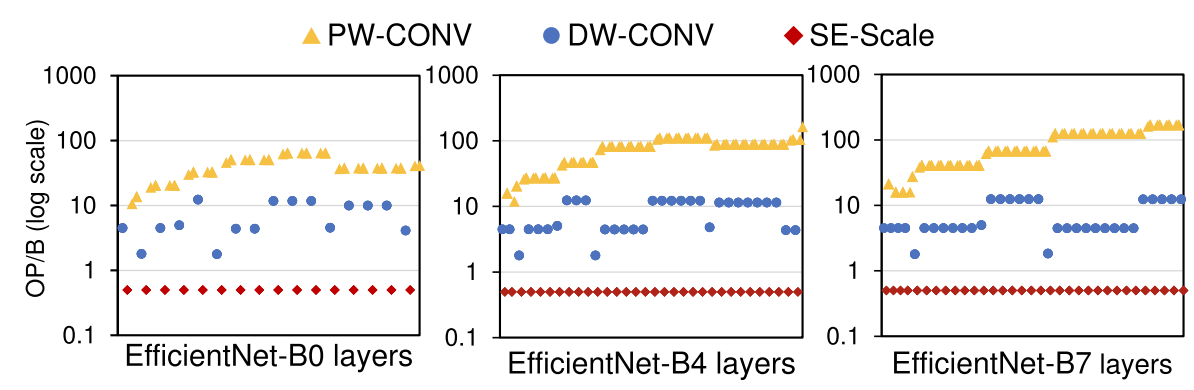

Fig. 5. Operations per byte (OP/B) of EfficientNet-B0/B4/B7 layers. The leftmost column represents the layer processing an input image. We only show PW-CONV, DW-CONV, and SE-Scale because they occupy most operations of the models. It is assumed that all three operations use 8-bit data type.

OFmaps of DW-CONV which is stored at UB or DRAM because all the Fmaps were used in the previous step. Last, the second PW-CONV follows SE-Scale.

The compositional characteristics of MBConv make the arithmetic intensity of EfficientNet fluctuate a lot. Figure 5 shows operations per byte $(O P / B)$ over the layers in EfficientNet-B0/B4/B7. The leftmost column in each graph represents the layer processing an input image. PW-CONV reuses an IFmap by OC times. Because EfficientNet populates more channels as it comes closer to the classification layer, OP/B increases from left to right on the graphs. DW-CONV produces an OFmap by using a single IFmap; therefore, EfficientNet's kernel sizes determine the degree of data reuse. EfficientNet uses $3 \times 3$ and $5 \times 5$ kernels. Similar to NORM and ACT (not shown in Figure 5), SE-Scale achieves a low OP/B due to the lack of Fmap data reuse in conducting element-wise operations. Even if the number of layers and functions differs by the network sizes (B0 being the smallest and B7 the largest), because OP/B fluctuates a lot and DW-CONV and element-wise operations appear frequently, CNN accelerators must be designed to perform both compute-and memory-intensive layers/functions equally well.

\subsection{Baseline CNN Acceleration Architecture}

TPU [30] and NVDLA [38] adopt systolic execution or its variants, achieving superior performance and energy efficiency than other architecture types [39] in performing CNN inference. SysAr fully utilizes the data reuse characteristics of CONV by operating $O\left(N^{2}\right)$ MACs with $O(N)$ on-chip memory bandwidth from UB in a systolic data flow manner (we assume the height $\left(H_{M U}\right)$ and width $\left(W_{M U}\right)$ of MU are both $\left.N\right)$. Because NVDLA similarly achieves high area and energy efficiency by multicasting IFmap elements to MACs, our proposed architecture can also be applied to NVDLA. In this paper, we use SysAr as a baseline.

SysAr consists of MU, UB, systolic data setup, weight FIFO, accumulator (ACC), main-memory controller, and VU that is including NORM/ACT/POOL units (see Figure 6(a)). MU is a systolic 


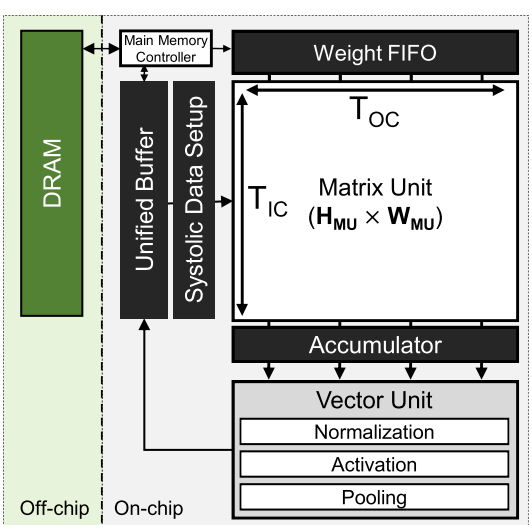

(a)
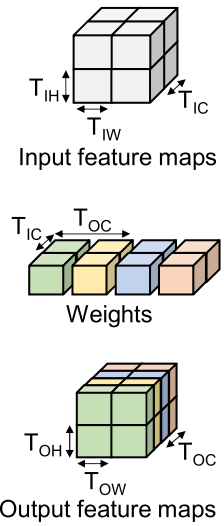

(b)

\begin{tabular}{|c|l|c|l|c|l|}
\hline $\mathrm{T}_{\mathrm{IH}}$ & Tiled IH & $\mathrm{T}_{\mathrm{IW}}$ & Tiled IW & $\mathrm{T}_{\mathrm{IC}}$ & Tiled IC \\
$\mathrm{T}_{\mathrm{OH}}$ & Tiled OH & $\mathrm{T}_{\mathrm{OW}}$ & Tiled OW & $\mathrm{T}_{\text {OC }}$ & Tiled OC \\
\hline
\end{tabular}

(c)

Fig. 6. (a) Baseline systolic-array architecture (SysAr). When SysAr computes a CONV layer, IC and OC are mapped to each row and column of MU [42, 43] without using im2col [9]. (b) Tiling features of ST/PW-CONV in SysAr. Tiling notations are listed in (c).

array, having MACs arranged in a two-dimensional form. We assume the weights and feature maps are 8-bit data. UB is an on-chip buffer that stores IFmaps and OFmaps. Through the systolic data setup, UB sends data to MU in a diagonal wavefront manner and saves the intermediate results. Weight FIFO temporarily stores a part of weights transferred from DRAM and sends them to the registers inside MU. ACC accumulates the partial sums calculated from MU and store them inside a buffer until partial sums become complete OFmap elements. NORM/ACT/POOL units perform element-wise operations on the 32-bit OFmap elements in a pipelined manner; the OFmap elements are converted into 8-bit values and transferred to UB.

Although MU targets GEMM operations, it also provides specialized hardware [42], algorithm [43], and instruction set architecture [30] for CONV. Therefore, SysAr can perform CONV without converting a 3D Fmap into a 2D one (im2col). Figure 6(b) shows the tiles of Fmaps and weights and their dimensions. Each row of MU takes one of $T_{I C}$ IFmap elements, while each column takes one of $T_{O C}$ weights. Each IFmap element propagates sequentially from left to right, and the output, which is a partial sum, propagates from top to bottom. Here we call each column a SysAr lane.

The Fmaps are divided into tiles if they do not fit in the on-chip storage (UB or ACC). ACC limits the size of an output tile $\left(T_{O W} \times T_{O H}\right)$ to the capacity of ACC divided by $T_{O C}$. Data traversal order is determined to maximize the data reuse of Fmaps and weights. Because the datapath from ACC to UB is uni-directional, each partial sum inside ACC must be a complete OFmap element. Thus, SysAr traverses IFmap and weights in all $I C$-, $K W$-, and $K H$-directions first.

\subsection{Challenges of Computing Memory-intensive CNN Layers}

Conventional CNN accelerators focus on compute-intensive ST-CONV and PW-CONV operations. They consider only limited types of memory-intensive operations that immediately follow CONV, such as NORM, ACT, and POOL. As opposed to ST-CONV and PW-CONV, DW-CONV does not reuse IFmap between OCs. Therefore, DW-CONV hardly exploits the broadcasting and systolic 


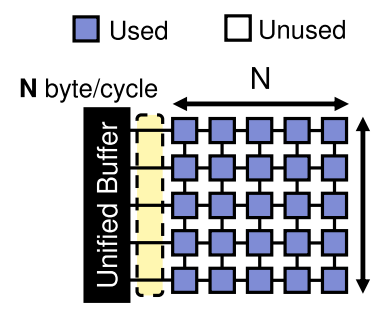

(a)
$i_{-i}^{-}$On-chip memory bandwidth

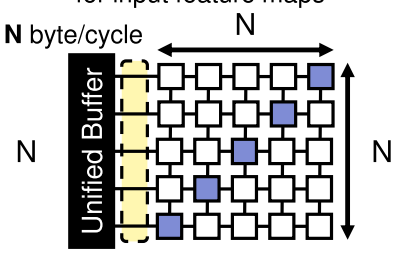

(b)

Fig. 7. MAC and on-chip memory bandwidth used inside of $M U$ when $N \times N$ Sys Ar computes CONV (assuming that IC and OC are bigger than N): the cases of (a) executing ST-CONV or PW-CONV and (b) executing DW-CONV.

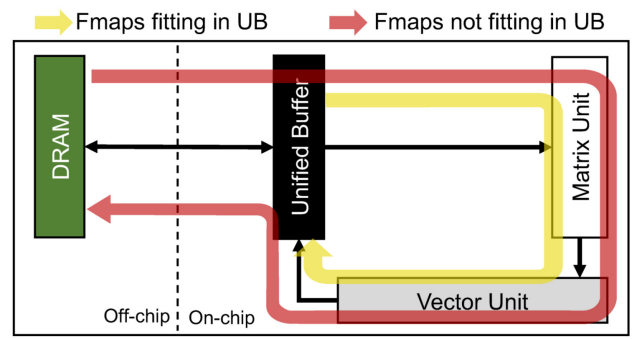

Fig. 8. Data movement patterns inside SysAr when performing SE-Scale. Fmaps can be read from either UB or DRAM, depending on their size. We focus on only Fmaps because they are much larger than the scale factors.

execution techniques mainly for IFmap reuse in conventional $\mathrm{CNN}$ accelerators. This characteristic of DW-CONV makes MACs in MU underutilized or requires more on-chip memory bandwidth to fully utilize MACs.

Figure 7(a) shows MACs in use and on-chip memory bandwidth usage when performing STCONV or PW-CONV in an $N \times N$ SysAr. At each cycle, the MACs in the leftmost column of MU takes $N$ IFmap elements transferred from the on-chip memory. The IFmap elements shift from left to right at each cycle, so each element is reused by $N$ weights. In a steady state where IFmap elements are distributed to all the MACs, $N$ B/cycle on-chip bandwidth is enough to utilize all the $N^{2}$ MACs. In contrast, DW-CONV requires each column of MACs to take IFmap elements from different OCs (see Figure 7(b)), not taking advantage of multicasting in systolic execution. Therefore, even if DW-CONV has a significantly smaller computational load compared to ST-CONV and PW-CONV, it takes a large portion of execution time due to low MAC utilization. SysAr can only supply the on-chip bandwidth of $N$ B/cycle even when processing DW-CONV. To fully utilize the $N \times N$ MACs, DW-CONV requires $O\left(N^{2}\right)$ of on-chip memory bandwidth, one of the most expensive resources in conventional $\mathrm{CNN}$ accelerators. ${ }^{1}$

In contrast to SysAr, Eyeriss-v2 [8] adopts a hierarchical mesh structure in its memory system, having its on-chip memory bandwidth high enough to fulfill the bandwidth requirement of DWCONV. This hierarchical structure exploits distributed SRAM for global buffer where each bank of the global buffer can transfer data simultaneously and connects several global buffer banks to local SRAM inside PEs in an all-to-all manner. However, a major drawback of this design is the

\footnotetext{
${ }^{1}$ The structural limitation of Edge TPU also makes it suffer from this problem, resulting in a much larger execution time than predicted by the number of operations [19]. To resolve this issue, Edge TPU replaces DW-CONV with more hardwarefriendly ST-CONV at a significantly increased computational cost.
} 
large area overhead as it needs to provide local SRAM for all the three data types: weights, IFmaps, and partial sum. As mentioned in [33], assuming an equal number of PEs, Eyeriss-v2 is more than twice as large as SysAr.

Conventional accelerators hide the execution time of NORM, ACT, and POOL by executing them within VU in a pipelined manner when they follow CONV. However, as opposed to these memoryintensive operations, such a pipelined execution is hardly applicable to SE-Scale that follows CONV because of the data dependency between SE-Scale and CONV (see Figure 4). SE-Scale requires a complete IFmap from the previous CONV to produce one single OFmap element.

Figure 8 shows the data movement pattern inside SysAr when performing SE-Scale. Depending on the size of Fmaps and UB, data should be read from either UB (yellow arrow) or DRAM (red arrow). We only depict the movement of Fmaps because Fmaps are much larger than the scale factors, whose number is the same as the number of channels.

When Fmaps fits in UB, SE-Scale performs multiplication after reading the Fmaps stored in UB. Because the datapath from UB to VU is uni-directional, Fmaps must go through MU to reach VU, and MU does not operate in the meantime, leading to severe underutilization of the MACs. Also, although SE-Scale requires much smaller computation than that of PW-CONV (150 250× in [52]), the execution time of SE-Scale is high because VU has $N \times$ fewer MACs than MU (see Figure 1). Even if MU handles SE-Scale to utilize its MACs, it takes the same time as in VU because the bandwidth of UB is limited to $O(N)$. When Fmaps do not fit into UB, off-chip memory bandwidth becomes a performance bottleneck because of the extra access to more bandwidth-hungry DRAM.

\section{MVP: A CNN ACCELERATOR WITH MATRIX, VECTOR, AND PROCESSING-NEAR-MEMORY UNITS}

\subsection{MVP Organization}

We propose a novel CNN accelerator architecture called $M V P$, which includes an MU, a VU for NORM/ACT (VU-NA) and for DW-CONV (VU-DW), and a processing-near-memory unit (PNMU) as shown in Figure 9(a). As opposed to the baseline SysAr architecture that suffers from poor performance in memory-intensive layers such as DW-CONV and SE, MVP is pragmatically designed to efficiently process both compute-intensive and memory-intensive CNN layers and functions with a minimal area overhead.

VU-DW takes the OFmaps from MU through VU-NA (processing PW-CONV, NORM, and $\mathrm{ACT}$ ) and performs DW-CONV in a pipelined manner (see Figure 9(b)). In case DW-CONV does not follow PW-CONV, the OFmaps bypass VU-DW and head to UB directly. Based on the key observation that a SysAr lane only processes an output channel, we place a depth-wise processing element (DWPE) per SysAr lane. Each DWPE consists of depth-wise input/weight buffer (DWIB/DWWB), $k$ depth-wise multipliers (DWMULs), an adder tree for partial reduction, and registers for accumulation. Each DWMUL takes the operands from DWIB and DWWB, multiplies them, and sends the output to the adder tree. DWIB is a buffer for IFmaps in DW-CONV; it consists of $k$ DWIB slices, each being mapped to a DWMUL. Each DWIB slice is a register file with a word size of 1-byte, allowing the DWMULs to access a row of DWIB SRAM cells simultaneously. DWWB is a buffer for weights in DW-CONV. Because the weights are reused with repeating but shuffled patterns to be aligned with the IFmap values to be multiplied at DWMUL, DWWB consists of a barrel shifter and a small register file. VU and UB are connected bi-directionally. VU-DW also covers the POOL (pooling) function because recent CNN models have relatively simple POOL operations such as global average pooling.

PNMU (Figure 9(c)) is a preprocessing unit which is placed near on-chip unified memory to execute simple element-wise operations conducted prior to CONV. These operations are pipelined 


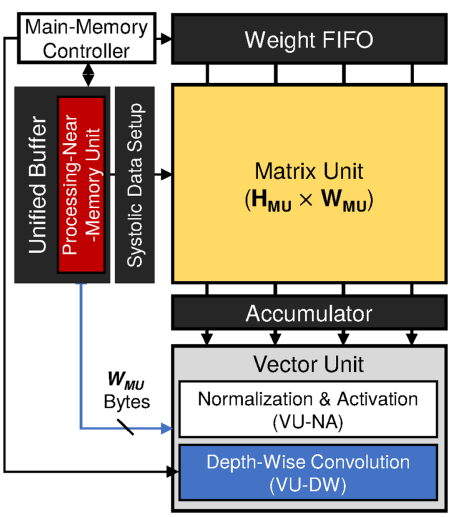

(a)

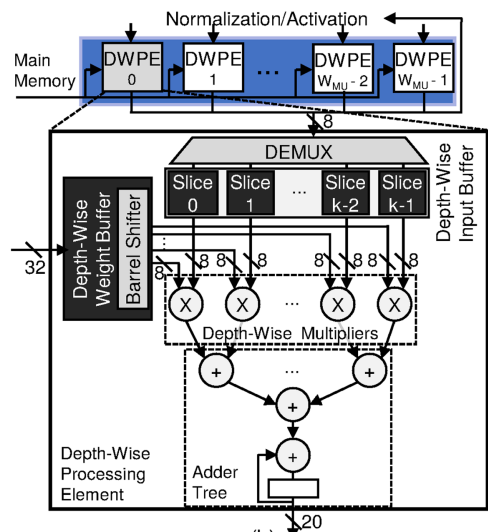

(b)

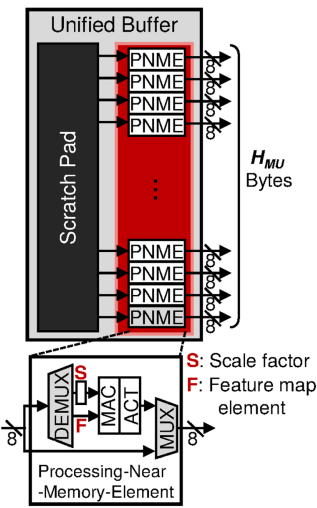

(c)

Fig. 9. (a) MVP architecture (the red and blue parts represent the units added to the baseline SysAr), (b) VU-DW, and (c) PNMU.

with the subsequent CONV, thereby hiding the execution time and saving memory accesses. PNMU includes $H_{M U}$ PNM elements (PNMEs), each placed between a row of UB and MU in an aligned manner. Each PNME consists of a DEMUX, a MAC, a comparator for ReLU, a MUX, and tiny registers holding scale factors used in scaling operations such as SE-Scale and NORM. DEMUX selects either a scale factor or an IFmap element. PNME only supports ReLU because it is a popular ACT function that is also used for MBConv [22, 26], and the logics for other ACT types take up a large area. MUX decides whether or not to use preprocessing.

\subsection{How Depth-wise Processing Element (DWPE) Operates}

We describe how DWPE operates by showing an example of a DW-CONV layer with stride 1 and with zero-padding: the sizes of inputs are $4 \times 4$ for IFmaps and $2 \times 2$ for weights (see Figure 10 (a)). Figure 10(b) presents the data movement in DWPE I/O at each cycle, while Figure 10(c) shows how DWPE operates internally in detail.

(1) VU-NA receives the OFmap elements of PW-CONV from MU and ACC, performs BN and ACT, and sends the resulting Fmap to DWPE per cycle at each lane. DWPE takes the IFmap element and stores it to DWIB. At cycle 0, DWIB stores the IFmap element in one of the four DWIB slices. The position of DWIB slice where an IFmap element is stored is determined by Equation (1).

$$
\text { Position of DWIB slice }=\left(\text { row }_{\text {IFmap }}+\text { column }_{\text {IFmap }} \times K H\right) \% D W M U L
$$

In the equation, the row IFmap and column $n_{\text {IF map }}$ are the positions of a single IFmap element on a 2-dimensional IFmap. For example, the row IFmap and column IFmap $_{\text {of an IFmap element numbered }}$ 0 are both 0 .

(2) At cycle 1, DWPE starts the first DW-CONV operation. The first CONV window requires a single IFmap element numbered 0 and three zero-padding elements. DWMULs only load an IFmap element numbered 0 from a DWIB slice for operation. DWMULs which need zero-padding elements do not work. Because weights are recycled for every OFmap element in an OFmap, the barrel shifter rearranges the weights by a shift distance according to Equation (2). If the zeropadding size is odd, a math symbol in the equation is a plus, and vice versa, the math symbol is a minus. The shift distance numbered 0 means that the weight elements are allocated to the DWMULs in the same way as storing IFmap elements to the DWIB slices as shown in cycle 0 . The equation of shift distance is similar to Equation (1); however, there are slight differences in 


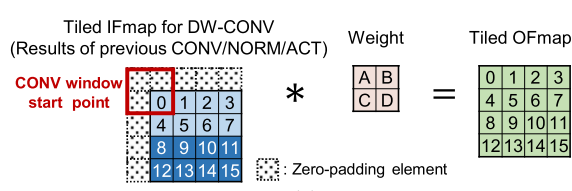

(a)

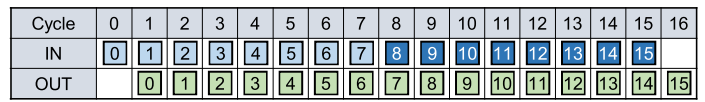

(b)

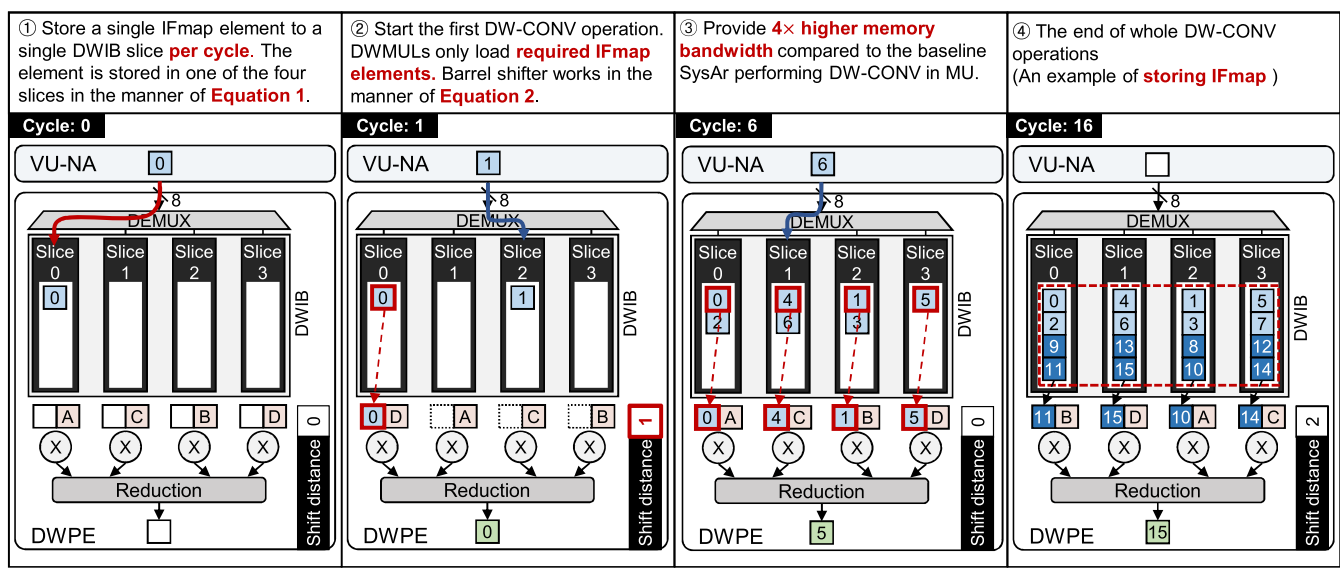

Fig. 10. An example of how DWPE operates when the number of DWMULs and DWIB slices is 4. Because the DWPEs perform the same operations on independent Fmaps, we describe an example for one DWPE. (a) An example of DW-CONV where IH and IW are the same as the tile size used in the previous PW-CONV $(4 \times 4$ in the example). (b) DWPE data in/out movement status at each cycle. The result of PW-CONV, NORM, followed by ACT, is transferred to DWPE every cycle, assuming that IC of the previous PW-CONV is smaller than $H_{M U}$ (weights are not shown for convenience of explanation). (c) DWPE operations in specific clock cycles. We assume that read/write operations of DWIB spend one cycle.

considering the OFmap element, ST, and the zero-padding size.

$$
\text { Shift distance }=\left[S T\left(\text { row }_{\text {OFmap }}+\text { column }_{\text {OFmap }} \times K H\right) \pm \text { padding }\right] \% D W M U L
$$

(3) From cycles 1 to 16, DWPE performs a convolution operation for every cycle. Compared to the baseline SysAr performing DW-CONV in MU, DWPE provides up to $4 \times$ higher memory bandwidth, owing to the 4 DWIB slices operating in parallel. To fully utilize all the DWIB slices at every cycle, each IFmap element in a CONV window should be placed in a proper DWIB slice. Cycle 6 is an example of fully utilizing $4 \times$ bandwidth. The CONV window requires the IFmap elements numbered $0,4,1$, and 5 : the four elements are distributed, in column-major order, into the first row of the four DWIB slices. DWPE loads the IFmap elements 0,4 , 1, and 5 from each DWIB slice, multiplies them with the corresponding weights, sums up the output to compute OFmap element 5. (4) This data access pattern is repeated until DW-CONV finishes at cycle 16.

Even when a stride is larger than 1, the DEMUX for storing IFmap in the DWIB slice works the same as the stride 1 . The pattern of loading IFmaps into DWMUL need not change. For example, when the stride is 2 , IFmaps are loaded in the same way as $6,8,14$, and 16 cycles of Figure 10(b). The barrel shifter does not change the position of weights when the window moves in the row direction; it only shifts weights by 2 when the window moves in the column direction. Thanks to such a straightforward access pattern, DWPE fully exploits the convolutional reuse on an IFmap, yet being flexible enough to support the various sizes of kernels and the number of DWMULs. 


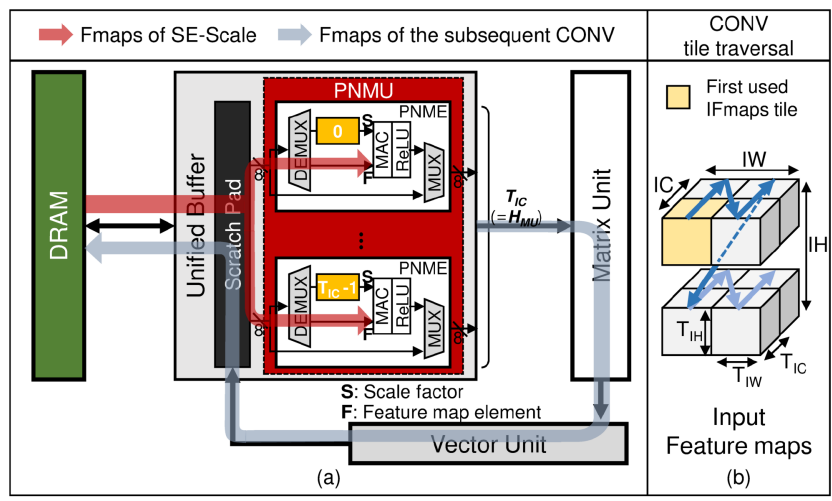

Fig. 11. Illustration of how PNMU operates. PNMU reads Fmaps from DRAM and performs SE-Scale and the subsequent CONV concurrently. (b) Tile traversal of the next CONV.

\subsection{How Processing-near-memory Unit (PNMU) Operates}

PNMU processes element-wise operations that commonly precede a CONV layer, such as NORM, $\mathrm{ACT}$, and SE-Scale. Figure 11 illustrates how PNMU processes SE-Scale. Here we describe an example of fetching IFmaps of SE-Scale from DRAM as it accompanies fetching from UB. First, scale factors required for SE-Scale are computed by VU, stored at UB, fetched from UB, and stored in the registers inside PNME. Each of $T_{I C}$ PNME holds its own scale factor for a single input channel (see Figure 11(a)). Then, PNMU multiplies the scale factors with a tile of Fmaps sized $T_{I W} \times T_{I H} \times T_{I C}$ Fmaps in an element-wise manner. The output of SE-Scale is directly fed into MU for the subsequent CONV.

After one Fmap tile is complete, one needs to determine which direction to traverse the Fmap tiles because it determines whether the scale factors are reused or not. As explained in Section 2.3, SysAr sends a complete OFmap element from ACC to VU. For that, SysAr traverses in IC-direction first (see Figure 11(b)); then, the tiles are traversed in $I W$ - and finally in $I H$-direction.

PNMU overlaps most of the execution time for SE-Scale with the subsequent CONV and saves memory access for SE-Scale, compared to the baseline SysAr. This design includes inevitable overheads, albeit minimal. Because any two IFmap tiles processed in a row have completely different input channels, the scale factors in the PNME registers should be updated for every IFmap tile. When the datapath between UB and PNME is busy updating the scale factors, it cannot feed the Fmap for SE-Scale and the subsequent CONV. However, once a scale factor is updated, it is reused by $T_{I W} \times T_{I H}$, amortizing the time overhead for the scale factor update to $\frac{1}{\left(T_{I W} \times T_{I H}\right)}$. For the CNN models taking input images with a resolution of $224 \times 224$, the smallest $T_{I W} \times T_{I H}$ is 49 ; limiting the time overhead to $2 \%$ (more details in Section 4.2 ).

\subsection{Overlapping the Operation of DW-CONV with PW-CONV}

VU-DW and MU can concurrently execute both PW-CONV and DW-CONV in a pipelined manner, resulting in additional speedup with the overlapped execution. Figure 12 shows timing diagrams of $\mathrm{PW}-\mathrm{CONV}$ and the following $\mathrm{DW}-\mathrm{CONV}$ with and without overlapping. Pipelining clearly reduces execution time. The degree of execution time saving depends on the time to produce an OFmap tile during PW-CONV and DW-CONV, defined as $\frac{T_{I W} \times T_{I H} \times I C}{H_{M U}}$ for PW-CONV and $\frac{T_{O W} \times T_{O H} \times K H \times K W}{D W M U L}$ for DW-CONV. Figure 12(b) shows the case when the processing time for an OFmap tile of PW-CONV is larger than that of DW-CONV. Then, the execution time on DW-CONV fully overlaps with PW-CONV. If the processing time for an OFmap tile in DW-CONV 


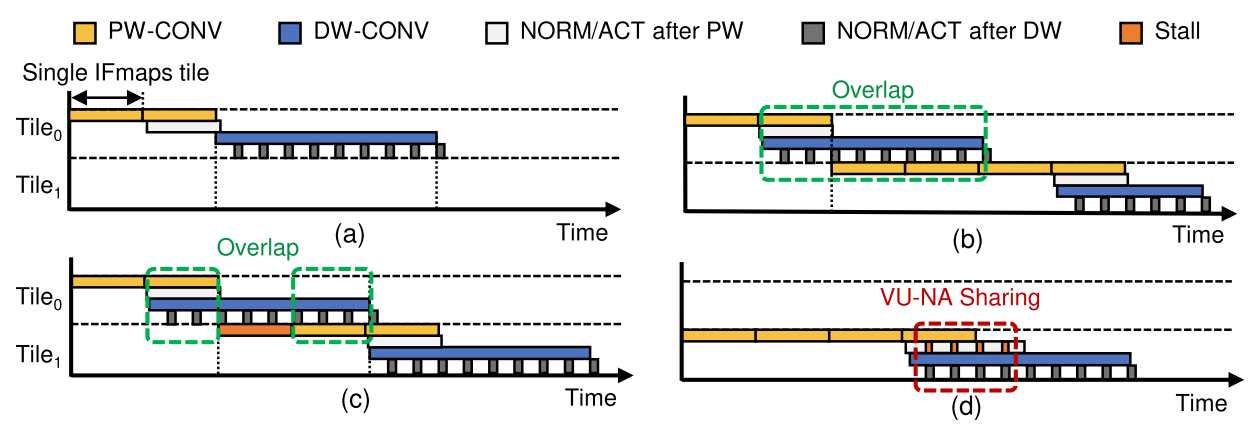

Fig. 12. Timing diagrams for processing PW-CONV followed by DW-CONV. X-axis represents time and $y$-axis represents operation sequences about multiple OFmap tiles. MU and VU process PW-CONV and DW-CONV (a) without pipeline, (b) with pipeline $\left(\right.$ Cycle $_{P W}>$ Cycle $\left._{D W}\right)$, (c) with pipeline $\left(\right.$ Cycle $_{P W}<$ Cycle $\left._{D W}\right)$, and (d) with sharing VU-NA.

is larger than that in PW-CONV (Figure 12(c)), there is a stall (shown as an orange bar) in MU, leading to less saving albeit still beneficial.

Meanwhile, the throughput requirement of VU-NA increases twice due to the concurrent processing. It is simple to duplicate VU-NA for DW-CONV and PW-CONV, but it requires $11 \%$ additional area (see Section 4.1). To avoid this, we share one VU-NA for both DW-CONV and PW-CONV through multiplexing (see Figure 12(d)). We present the performance overhead due to sharing VU-NA in Section 4.2.

\subsection{Considerations for Designing DWIB}

Because the DWIB size affects the execution time and energy of both DW-CONV and PW-CONV and the area of MVP, it must be carefully designed. PW-CONV can suffer from slowdown and more energy consumption due to the additional DRAM memory access if DWIB size is too small. PWCONV might experience more frequent DRAM accesses due to weight update when concurrently executing PW-CONV and DW-CONV due to a smaller PW-CONV tile size compared to the nonoverlapped case [35]

DWIB also affects DW-CONV performance. Because DW-CONV uses a kernel size of $3 \times 3$ or larger, the edge part of the previously used IFmap tile is required (we call it an edge IFmap). All the edge IFmaps are stored in UB and must be moved to DWIB through a bidirectional path between UB and VU. However, because the same path is occasionally used for sending the OFmaps of DW-CONV to UB, contention can occur. The size of edge IFmaps increases in proportion to the number of the DW-CONV IFmap tiles, which is closely related to DWIB. We quantify overhead by the contention (Section 4.2), and evaluate MVP performance and energy changes according to the DWIB size (Section 4.4).

\section{EVALUATION}

We quantified the benefits of MVP architecture over SysAr on execution time, energy, and area. Then we explored the various design spaces of MVP, such as DWIB size and the number of DWMULs. We also demonstrated the MVP's performance improvement across various SysAr configurations with different MU dimensions and the size of UB and ACC.

\subsection{Experimental Setup}

SysAr is the baseline architecture for evaluation. We set the baseline to have an MU with $64 \times 64$ MACs, $512 \mathrm{~KB}$ UB, and $128 \mathrm{~KB}$ ACC buffer (see Table 2). This is the best-performing configuration 
Table 2. SysAr, VU-DW, PNMU, and DRAM Specifications

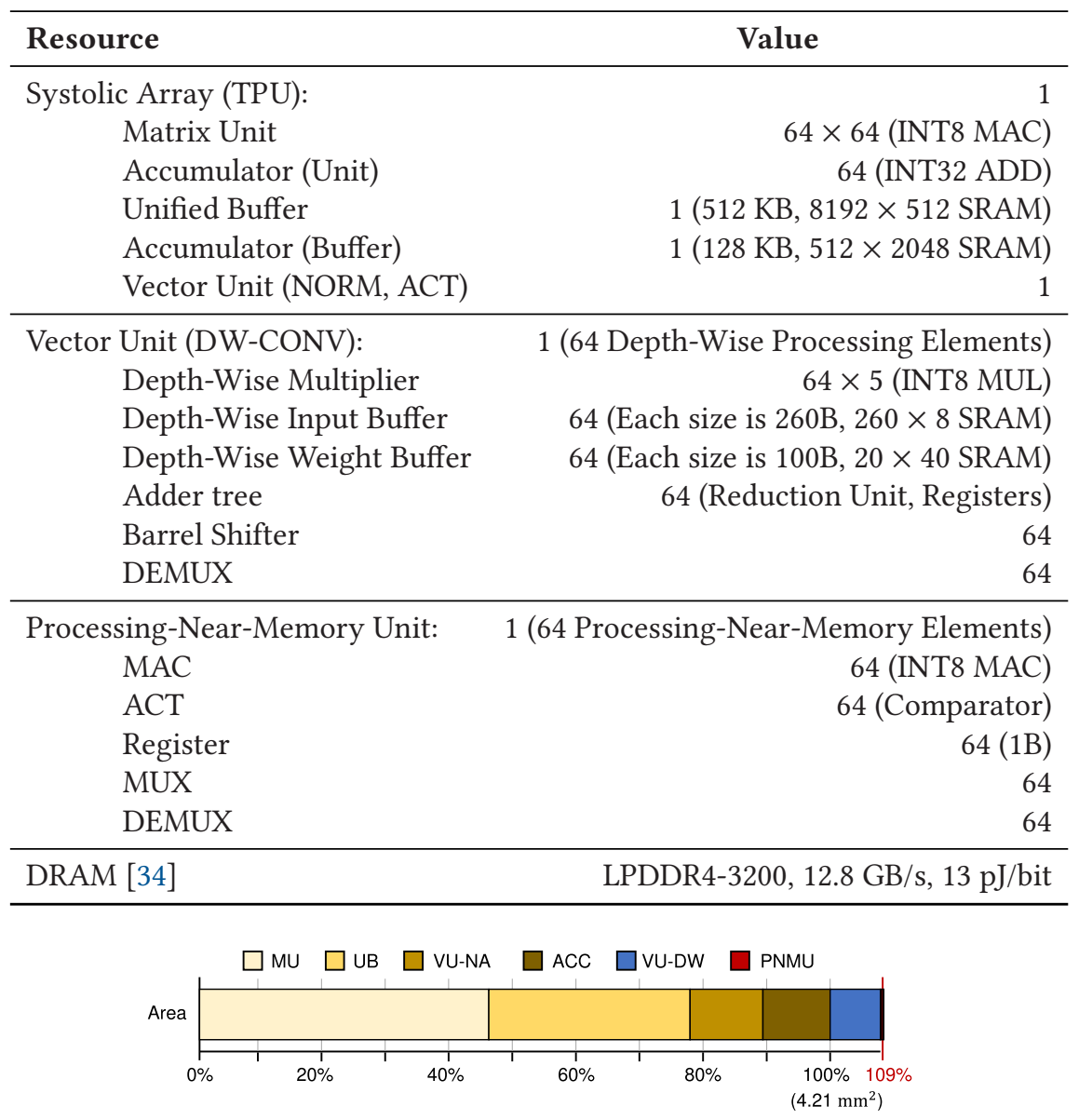

Fig. 13. MVP area breakdown.

on ResNet-50 and VGG-16 models based on $E D A^{2} P$ (Energy Delay Area ${ }^{2}$ Product) metric [36]. To get a reasonable SysAr configuration for ST/PW-CONV, we used ResNet-50 and VGG-16 because they are the most well-known CNN models among the ones not using DW-CONV and SE. VU-DW consists of $64\left(=W_{M U}\right)$ DWPEs, each including five 8-bit DWMULs, a 260B DWIB with five slices, a 100B DWWB, and an adder tree. PNMU has $64\left(=H_{M U}\right)$ PNMEs, each including DEMUX, 8-bit MAC unit, ACT unit, MUX, and register. Compared to SysAr, MVP adds an area overhead of 9\% where VU-DW and PNMU incur $8 \%$ and $1 \%$, respectively (see Figure 13 ).

We used Timeloop [39] for evaluation. Timeloop is a simulator for evaluating CNN accelerators. Timeloop has two main components: a model to provide performance, area, and energy projections of hardware components and a mapper to find the optimal mapping of tiling and loop order of any layers on the targeted architecture. Timeloop requires an ERT (energy reference table) and an ART (area reference table) that contain energy and area values for each hardware component. We used a custom ERT and ART, whose values were calculated as follows. We synthesized logic components such as multipliers and MACs based on TSMC 40nm technology and evaluated the SRAM component of MVP using CACTI [53]. We set the clock frequency and data precision to 
be $500 \mathrm{MHz}$ and 8-bit, respectively. We modified Timeloop to support DW-CONV and SE layers. The mapper of the existing Timeloop officially supports the layer shape only for CONV. However, Timeloop elaborates that they can support FC by modifying the layer shape of CONV (IH, IW, OW, $\mathrm{OH}, \mathrm{KW}$, and $\mathrm{KH}$ are fixed to 1, whereas only IC and OC are changed) as mentioned in [39]. We modified the mapper similarly to FC, considering that DW-CONV is a variant of CONV and SE consists of two FCs and one element-wise multiplication layer. Also, we modified the model to add architectures such as VU-DW and PNMU that are not supported by Timeloop. We validated VU-DW and PNMU by using the synthesis results of our RTL design.

We used EfficientNet-B0/B4/B7, MnasNet, and MobileNet-V1/V2 as our target CNN models. Although MobileNet-V1 does not include MBConvs, MVP can support the model as it has a similar layer pattern where $D W$-CONV follows $P W-C O N V$ and multiple depth-wise separable CONVs are repeated. We didn't evaluate NoisyStudent, FixEfficientNet, and Meta Pseudo Labels because they exploit the same MBConv and thus have a similar structure to EfficientNet.

\subsection{Performance and Energy Evaluation}

We demonstrate the performance and energy efficiency of MVP for the target CNN models. We quantify the performance improvements in detail by incrementally applying VU-DW, the overlapped execution of PW-CONV and DW-CONV, and PNMU. We verify that there is no significant difference between SysAr and MVP when running ResNet-50 and VGG-16 as MVP can operate like SysAr by bypassing the added units and the additional wiring cost is negligible. We make the following key observations.

MVP significantly reduces the execution time of DW-CONV and SE-Scale. Figure 14(a) shows the relative execution time of MVP on the latest CNN models. ${ }^{2}$ First, when VU-DW is applied without overlapping, the execution time reduces by $51 \%$ on average, mostly attributed to saving time in processing DW-CONV. VU-DW exploits its high internal memory bandwidth supported by DWIB; and it utilizes up to $5 \times$ more MACs than SysAr, which utilizes only $N$ MACs (as shown in Figure 7), thereby reducing the execution time of DW-CONV by $78 \%$ on average. Also, VU-DW alleviates the off-chip memory bandwidth bottleneck by forwarding the OFmap elements of PW-CONV from ACC directly into VU-DW (through VU-NA) and thus removing the accesses to DRAM. The processing speed of PW-CONV also improves, especially for larger models such as EfficientNetB7. Second, with the overlapped execution of PW-CONV and DW-CONV, we further observe an average speedup of 5\%. Last, except for MobileNet-V1/V2, which do not include SE-Scale, PNMU improves the processing speed additionally by $8 \%$ on average. As mentioned in Section 3.3, PNMU removes memory accesses to UB and DRAM while executing SE-Scale. The speedup is higher for models with large Fmap sizes. Overall, the total execution time is improved by $61 \%$ on average.

MVP significantly saves energy consumption primarily by reducing the DRAM memory accesses. Figure 14(b) shows the relative energy consumption of MVP over the baseline SysAr. When VUDW is applied without overlapping, total energy consumption is reduced by $41 \%$, where the energy reduction in DRAM accesses is dominant. It is because VU-DW eliminates the DRAM accesses that were necessary for delivering the intermediate Fmaps between PW-CONV and DW-CONV before. VU-DW also reduces the energy consumption of UB in two ways: (1) by eliminating the memory accesses on the intermediate Fmaps between PW-CONV and DW-CONV, and (2) by reducing the repeated memory accesses on the same Fmap elements through exploiting the convolutional reuse stated in Section 3.2. The reduced energy consumption in ACC, on the other hand, attributes to the on-the-fly accumulation of partial sums through the adder tree in DWPE; the partial sums are

\footnotetext{
${ }^{2}$ We point out that the SysAr configured for evaluation has a similar performance to EdgeTPU [16]: the execution time of MobileNet-V2 with SysAr (1.4ms) is similar to that with EdgeTPU (2.6ms)
} 


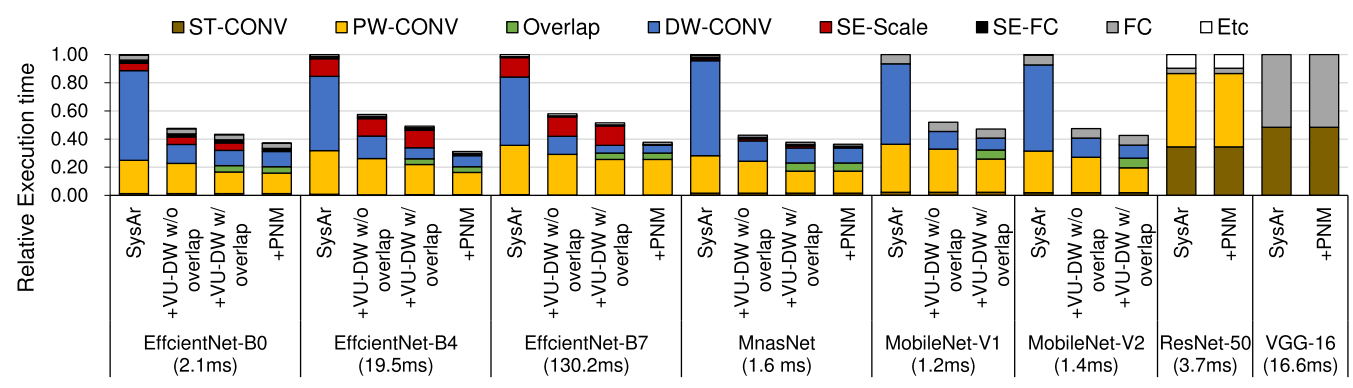

(a)

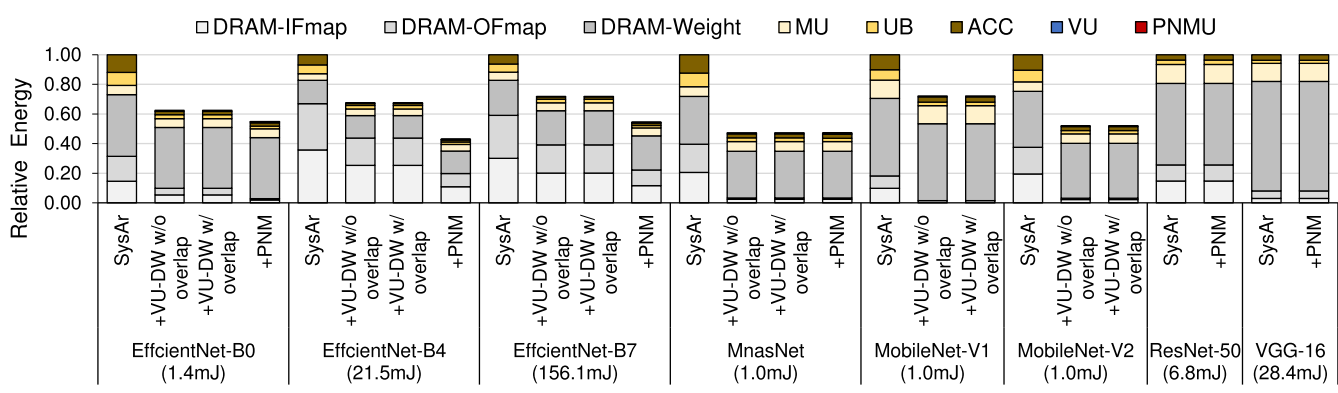

(b)

Fig. 14. (a) Relative execution time and (b) relative energy consumption of MVP over SysAr by incrementally applying VU-DW, the overlapped execution between PW-CONV and DW-CONV (shown as overlap), and PNMU. The absolute execution time and energy comsumption of the baseline SysAr is shown below the name of each CNN model. The execution time of MobileNet-V2 with SysAr is in a similar ball park to the reported execution time of EdgeTPU [16] (2.6ms), which has the same peak FLOPS as the evaluated SysAr.

accessed through small registers instead of large memory in ACC while processing DW-CONV. Meanwhile, there is little energy savings by overlapping because it affects only the timing of processing each layer. PNMU further reduces the energy consumption by $9 \%$, owing to the elimination of DRAM and UB traffic in SE-Scale. Putting it all together, MVP reduces energy consumption by $47 \%$ in total.

We further quantify the performance overheads of MVP due to the limited DWIB capacity and the time-division multiplexing of VU-NA. When an IFmap of DW-CONV does not fit into the limited DWIB capacity, as explained in Section 3.5, we fetch the edge IFmaps from UB, which incurs small performance overhead. Figure 15 shows that the overhead of fetching edge IFmaps (shown as Edge overhead) is $4 \%$ for EfficientNet-B0. This overhead happens only in close-to-input BBs whose IFmaps are large. In contrast, Share overhead (performance overhead due to the contention by VUNA sharing) and Scale overhead (performance overhead caused by the contention when SE-scale is performed in PNMU) have little effect on execution time. The Share overhead is low because the frequency of traffic requested to the VU-NA is low. VU-NA is utilized when CONV produces OFmaps. In the case of PW-CONV, VU-NA requests occur in a period of $\frac{I C}{T_{I C}}$ cycles, as mentioned in Section 2.3. DW-CONV uses VU-NA in a period of two or five cycles for the kernel sizes of $3 \times 3$ and $5 \times 5$, respectively, when using 5 DWMULs per DWPE. Therefore, as VU-NA requests from PW-CONV and DW-CONV occur intermittently, MVP can operate with little overhead using a single VU-NA through time multiplexing. The Scale overhead is negligible because the period of reading scale factors is much less than that of Fmaps, as mentioned in Section 3.3. Overall, the total performance overhead for the target $\mathrm{CNN}$ models is $2.6 \%$ on average. 


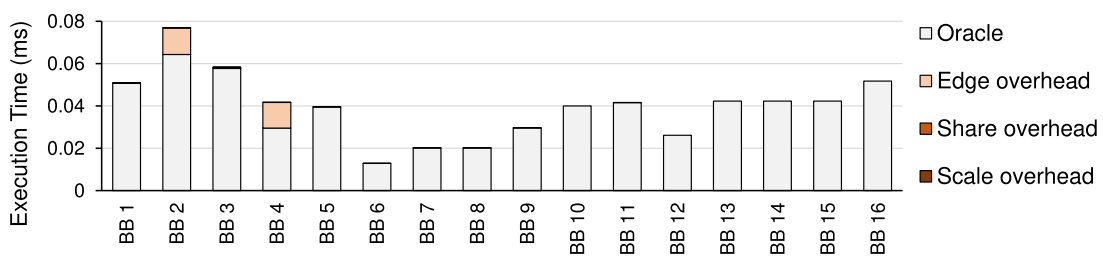

Fig. 15. Execution time of EfficientNet-B0 over BBs. Edge overhead means performance overhead due to the datapath contention caused by the edge IFmaps (Section 3.5). Share overhead means performance overhead due to the resource contention by VU-NA sharing (Section 3.4). Scale overhead means performance overhead due to the datapath contention when SE-scale is performed in PNMU (Section 3.3). Oracle is an execution time when all three overheads do not exist.

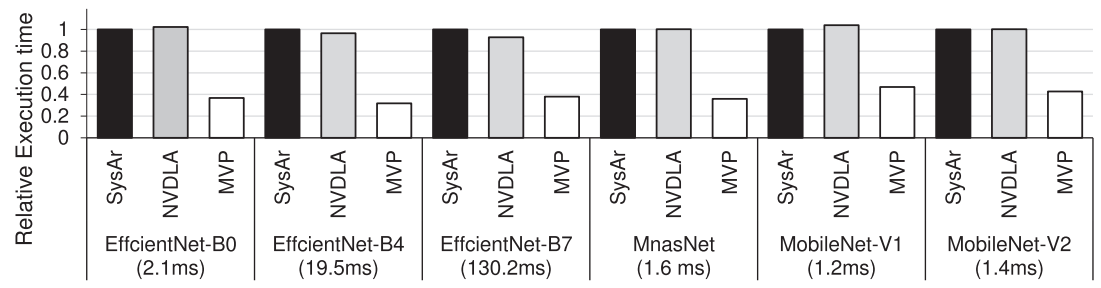

(a)

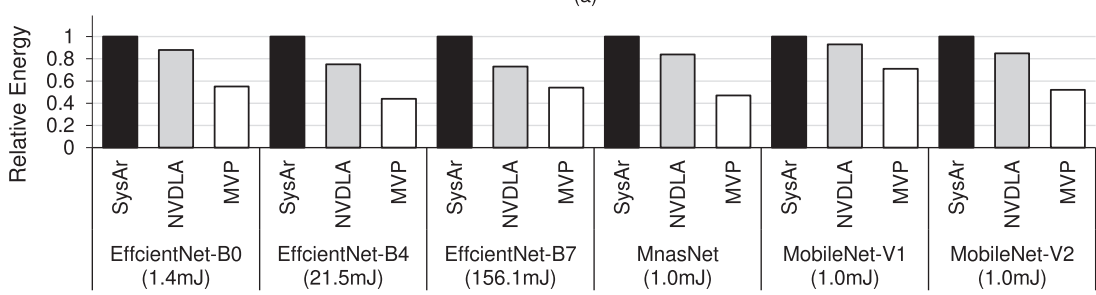

(b)

Fig. 16. (a) Relative execution and (b) relative energy of three accelerators to compare MVP with NVDLA. The absolute execution time and energy consumption of the baseline SysAr is shown below the name of each CNN model.

\subsection{Comparing MVP with NVDLA}

We further compare the execution time and energy consumption of MVP with those of NVDLA. NVDLA consists of a convolution MAC unit, a convolution buffer, and a convolution accumulator. The convolution MAC unit consists of $H_{M U}$ MAC cells, which include $W_{M U}$ multipliers and an adder tree. A single MAC cell takes one of $T_{I C}$ IFmap elements, while $H_{M U}$ MAC cells take one of $T_{O C}$ weight elements. The convolution buffer is an on-chip buffer that stores IFmaps and weights. The convolution accumulator stores the partial sums that are computed by the MAC cells until they become a complete OFmap element. We set NVDLA as a $64 \times 64$ convolution MAC unit, a $512 \mathrm{~KB}$ convolution buffer, and a $128 \mathrm{~KB}$ convolution accumulator similar to the baseline SysAr $(\simeq \mathrm{TPU})$.

MVP reduces execution time compared to NVDLA, and the reduction rate is almost the same as that of SysAr. Figure 16(a) shows the relative execution time of SysAr, NVDLA, and MVP for the latest CNN models. NVDLA is similar to SysAr in how to allocate the IFmap, weights, and OFmap tiles to the convolution MAC unit, and the OFmap element can be moved out of the convolution accumulator when partial sums become a complete value. Therefore, NVDLA has less than $2 \%$ of 


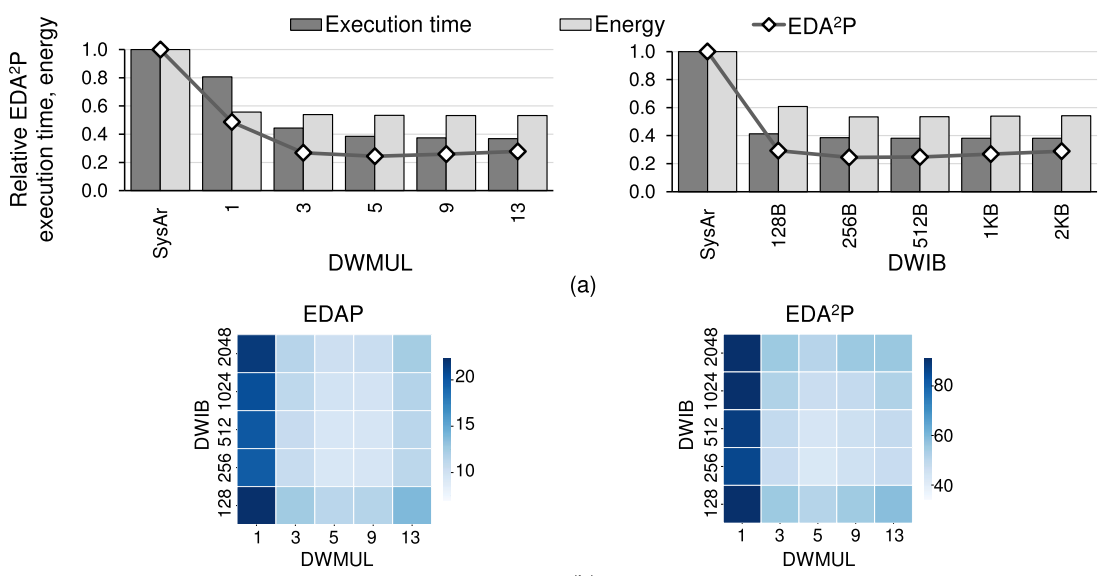

(b)

Fig. 17. (a) Execution time, Energy, and $E D A^{2} P$ when the number of DWMULs is varied for a 256B DWIB size (Left side) when the DWIB size is varied for 5 DWMULs (Right side). (b) $E D A P$ and $E D A^{2} P$ heatmap by the number of DWMULs and the DWIB size (the lower, the better [36]).

MAC utilization when processing DW-CONV as mentioned in Section 2.5. Overall, MVP reduces the execution time for the entire $\mathrm{CNN}$ by $60 \%$ on average.

$M V P$ reduces energy consumption compared to NVDLA, but the reduction rate is less than that of SysAr. In the CNN models where PW-CONV, NORM, ACT, and DW-CONV are repeated, MVP executes PW-CONV, NORM, and ACT from MU through VU-NA and performs DW-CONV in VUDW by taking results directly from VU-NA. In contrast, NVDLA must access UB or DRAM after processing PW-CONV, NORM, and ACT. Thus, MVP reduces energy by $34 \%$ on average compared to NVDLA as shown in Figure 16(b). However, the energy consumption of NVDLA is smaller than that of SysAr. Because NVDLA reuses weights in the on-chip memory, it reads fewer weights from DRAM. In addition, the gap between the two accelerators is getting larger as the image resolution of the $\mathrm{CNN}$ model increases because the accelerator has to read weights repeatedly from DRAM or UB. Therefore, NVDLA saves energy consumption by $12 \%$ on average with $224 \times 224$ resolution (EfficientNet-B0, MnasNet, MobileNet-V1, and MobileNet-V2) and by $26 \%$ on average with $300 \times 300$ and $600 \times 600$ resolution (EfficientNet-B4 and EfficientNet-B7) compared to SysAr.

\subsection{Exploring the Design Space of MVP Architecture}

Increasing design parameters of MVP, such as the DWIB size and the number of DWMULs, can improve performance and energy efficiency but worsen area efficiency. We explore the design space of MVP and find optimal design parameters for the given SysAr configuration by using EDAP and $E D A^{2} P$ metrics, where the latter puts more weights on area efficiency. We use the geometric mean of all target $\mathrm{CNN}$ models to show simple results.

The number of DWMULs significantly affects the execution time. The left side of Figure 17(a) shows the execution time, energy, and $E D A^{2} P$ of MVP for a various number of DWMULs with $256 \mathrm{~B}$ DWIB. Even only adding one DWMUL to SysAr greatly reduces the execution time and energy consumption; it gives the same internal memory bandwidth as SysAr but eliminates DRAM and UB accesses between PW-CONV and DW-CONV, and overlaps the executions. PNMU additionally removes the DRAM and UB access, and hides the execution. Increasing the number of DWMULs improves the processing speed by utilizing more ALUs and memory bandwidth until the performance is saturated when the number of DWMULs is 5 . The performance saturates because the 


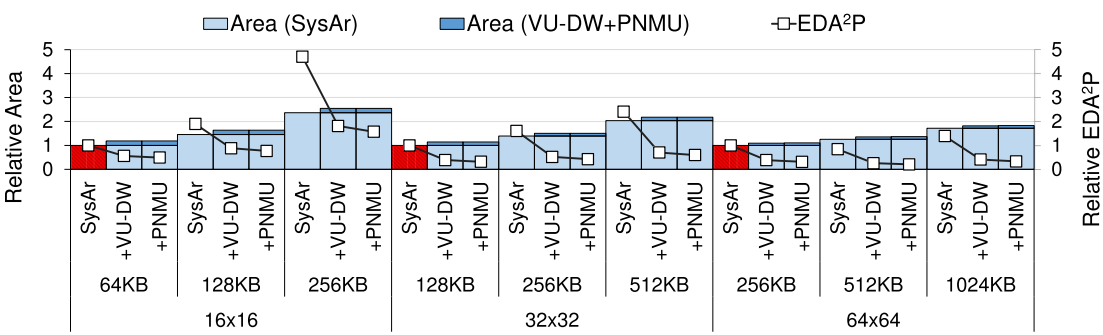

Fig. 18. Area and $E D A^{2} P$ of MVP while varying $M U, U B$, and ACC parameters. The outermost category on the $\mathrm{x}$-axis represents the $\mathrm{MU}$ size and the inner category represents the UB size. We select a candidate configuration that fits $U B$ and ACC capacity in proportion to the change in MU size and set ACC as one fourth of the UB size. We obtain the design parameters of MVP for each SysAr configuration by using the design space exploration conducted in Section 4.4. $E D A^{2} P$ values are calculated as the geometric mean for the target $\mathrm{CNN}$ models. Both $E D A^{2} P$ and area are expressed as relative values based on each SysAr configuration, which has the smallest UB in each MU size (colored red).

execution time of PW-CONV remains unchanged after adding one DWMUL and thus dominates. In contrast, populating more than 1 DWMUL has little impact on total energy consumption. This is because the number of on-/off-chip memory accesses, which dominate energy consumption, stays the same after adding one DWMUL. Finally, $E D A^{2} P$ decreases until 5 DWMULs and then starts increasing because of the growing area overhead.

Even MVP with small DWIBs outperforms SysAr, with high energy efficiency. Increasing the DWIB size has pros and cons. It enables DWIB to accommodate larger DW-CONV tiles, reducing both UB accesses on edge IFmaps and repeated DRAM accesses on the same weights of PW-CONV. However, the energy per memory access grows as DWIB becomes larger. The right side of Figure 17 (a) shows the execution time, energy, and $E D A^{2} P$ with various sizes of DWIBs. DWIB of 128B size causes repeated memory accesses to DRAM for fetching weights of $\mathrm{PW}-\mathrm{CONV}$, diluting the energy savings of MVP. Increasing DWIB over 256B worsens the energy efficiency because the energy per memory access increases. In contrast, an Fmap tile already at 256B becomes large enough to eliminate the repeated DRAM accesses on the same weights. The energy spent by the edge traffic is below $0.3 \%$ on average, which is negligible. Because of these reasons, the optimal size minimizing the energy consumption for DWIB is 256B. Meanwhile, as the DWIB size increases, the area increases, and the area efficiency worsens. As the DWIB size increases, it has an area overhead up to $18 \%$, and $E D A^{2} P$ increases as well.

We choose the parameters for MVP, the DWIB size and the number of DWMULs, for design space exploration based on the $E D A P$ and $E D A^{2} P$ metrics (see Figure 17(b)). In general, increasing each of the two parameters leads to better performance while increasing area and energy overhead. We find that the point with 5 DWMULs and 256B DWIB gives the best (lowest) EDAP and $E D A^{2} P$; we set them as default parameter values for MVP.

\subsection{Evaluating MVP with Various SysAr Configurations}

The proposed MVP is flexible enough to improve both the performance and energy efficiency across a wide range of configurations. We quantify the performance improvement of MVP with various sizes of MU, UB, and ACC. As shown in Figure 18, all the configurations show significant $E D A^{2} P$ improvement with VU-DW, which is further increased by PNMU, with only a small area overhead. In conclusion, MVP reduces the $E D A^{2} P$ by at least $51 \%$ and up to $76 \%$ for a range of configurations of SysAr. 


\section{RELATED WORK}

Conventional DNN inference accelerators: Accelerators often use 2D MAC array structure to accelerate $\mathrm{CNN}$ inference by using their own dataflows in exploiting the data reuse characteristics of ST/PW-CONV. NVDLA [38] consists of $T_{O C} 1 \mathrm{D}$ vector engines that process $T_{I C}$ MAC operations. NVDLA does not use systolic execution; however, dataflow, data mapping, and UB bandwidth are similar to TPU. Because NVDLA suffers from the same challenges as TPU as mentioned in Section 2.5, it also processes DW-CONV inefficiently. ShiDianNao [13] specializes in reusing IFmaps between sliding windows. Although ShiDianNao is good for processing ST and DW-CONV with a large kernel size, it cannot process PW-CONV efficiently because it does not utilize OC parallelism as mentioned in [32]. As opposed to SysAr, TPU v3 [29] changes VU structure to accelerate less arithmetically intensive operations such as inverse-square-root of BN while training, albeit not elaborating on details about processing DW-CONV in the modified VU. In addition, as TPU $\mathrm{v} 4$ [28] reuses hardware designs of TPU v3 except for several components such as on-chip memory capacity, on-chip interconnect, and DMA, the VU of TPU v4 is the same structure as that of TPU v3. There have been processing-near-DRAM studies $[10,14,31]$ to provide high off-chip memory bandwidth during inference. Because $[10,14]$ use dataflow architecture such as Eyeriss v1 [7] and systolic array, they still do not process DW-CONV efficiently. In contrast, [31] has advantages for memory-intensive operations but has weaknesses for compute-intensive ST-CONV operations.

Prior works supporting both ST- and DW-CONV: Previous architectural solutions have been mainly proposed to process both ST- and DW-CONV in an MU. Eyeriss v2 [8] provides flexibility in moving data from UB to PE by composing the memory hierarchy in two stages, mesh and all-to-all, so that both types of CONV could be processed while maintaining the existing dataflow. However, a major drawback of this design is the large area overhead as it needs to provide local SRAM for all the three data types. [4, 60,62] make hardware reconfigurable to process two types of CONV in an MU by changing dataflow and data mapping according to the CONV type. These solutions can accelerate DW-CONV using the existing MU, but require $O\left(N^{2}\right)$ UB bandwidth directly to the $\mathrm{MU}$ and incur a huge amount of wiring cost, one of the most expensive resources in conventional CNN accelerators. In-memory accelerators $[1,2]$ that accelerate DW-CONV have been proposed, albeit they target CNN models using binarized DW-CONV. [57] proposes a scheduling method that reduces off-chip access by fusing three consecutive layers inside the MBConv. However, it does not deal with the under-utilization when conventional accelerators operate DW-CONV. HPIPE [20] generates a customized dataflow inference accelerator for FPGA by considering layer information of the targeted CNN model and hardware resources such as DSP and BRAM. HPIPE minimizes unnecessary off-chip memory access and computation by processing multiple layers in a pipelined manner and by skipping zero activation and weight. HPIPE is efficient where the user processes a single CNN model or hardware changes are flexible.

\section{CONCLUSION}

We have proposed an MVP architecture composed of Matrix (MU), Vector (VU), and Processingnear-memory units (PNMU) to effectively accelerate the inference of the latest CNN models. As opposed to the conventional area-efficient accelerators which mainly focus on convolution (CONV) with high arithmetic intensity (e.g., point-wise CONV), MVP effectively supports both compute- and memory-intensive layers. We significantly reduce the execution time of depth-wise CONV by adding efficient processing elements for depth-wise CONV (called DWPE) with high-bandwidth low-capacity register files (called DWIB) in the VU of the baseline systolic-array architecture to complement the low-bandwidth high-capacity unified buffer (UB). This design enables the pipelined execution of point-wise CONV and depth-wise CONV, further improving the 
performance. Also, a lightweight PNMU added to UB substantially reduces the execution time of squeeze and excitation, a critical building block of the latest CNN models. Our evaluation shows that MVP improves the performance of EfficientNet-B0/B4/B7, MnasNet, and MobileNet-V1/V2 by $2.6 \times$ and total energy by $47 \%$ on average while incurring only a $9 \%$ area overhead compared to the baseline.

\section{REFERENCES}

[1] Shaahin Anginzi, Zhezhi He, and Deliang Fan. 2018. DIMA: A depthwise CNN in-memory accelerator. In Proceedings of the IEEE/ACM International Conference on Computer-Aided Design (ICCAD). https://doi.org/10.1145/3240765.3240799

[2] Shaahin Anginzi, Zhezhi He, Adnan Siraj Rakin, and Deliang Fan. 2018. CMP-PIM: An energy-efficient comparatorbased processing-in-memory neural network accelerator. In Proceedings of the ACM/ESDA/IEEE Design Automation Conference (DAC). https://doi.org/10.1109/DAC.2018.8465860

[3] Apple. 2017. On-device Deep Neural Network for Face Detection. https://machinelearning.apple.com/research/facedetection.

[4] Lin Bai, Yiming Zhao, and Xinming Huang. 2018. A CNN accelerator on FPGA using depthwise separable convolution. IEEE Transactions on Circuits and Systems II (2018). https://doi.org/10.1109/TCSII.2018.2865896

[5] Pete Bannon, Ganesh Venkataramanan, Debjit Das Sarma, and Emil Talpes. 2019. Computer and redundancy solution for the full self-driving computer. In IEEE Hot Chips Symposium (HCS). https://doi.org/10.1109/HOTCHIPS.2019. 8875645

[6] Tianshi Chen, Zidong Du, Ninghui Sun, Jia Wang, Chengyong Wu, Yunji Chen, and Olivier Temam. 2014. DianNao: A small-footprint high-throughput accelerator for ubiquitous machine-learning. In Proceedings of the 19th International Conference on Architectural Support for Programming Languages and Operating Systems (ASPLOS). https://doi.org/10. 1145/2541940.2541967

[7] Yu-Hsin Chen, Tushar Krishna, Joel Emer, and Vivienne Sze. 2016. Eyeriss: An energy-efficient reconfigurable accelerator for deep convolutional neural networks. In IEEE International Solid-State Circuits Conference (ISSCC). https://doi.org/10.1109/ISSCC.2016.7418007

[8] Yu-Hsin Chen, Tien-Ju Yang, Joel Emer, and Vivienne Sze. 2019. Eyeriss v2: A flexible accelerator for emerging deep neural networks on mobile devices. IEEE Journal on Emerging and Selected Topics in Circuits and Systems 9, 2 (2019), 292-308. https://doi.org/10.1109/JETCAS.2019.2910232

[9] Sharan Chetlur, Cliff Woolley, Philippe Vandermersch, Jonathan Cohen, John Tran, Bryan Catanzaro, and Evan Shelhamer. 2014. cuDNN: Efficient primitives for deep learning. (2014). arXiv:1410.0759 https://arxiv.org/abs/1410.0759.

[10] Seunghwan Cho, Haerang Choi, Eunhyeok Park, Hyunsung Shin, and Sungjoo Yoo. 2020. McDRAM v2: In-dynamic random access memory systolic array accelerator to address the large model problem in deep neural networks on the edge. IEEE Access 8 (2020), 135223-135243. https://doi.org/10.1109/ACCESS.2020.3011265

[11] François Chollet. 2017. Xception: Deep learning with depthwise separable convolutions. In Proceedings of the IEEE Conference on Computer Vision and Pattern Recognition (CVPR). https://doi.org/10.1109/CVPR.2017.195

[12] Xiaohan Ding, Xiangyu Zhang, Ningning Ma, Jungong Han, Guiguang Ding, and Jian Sun. 2021. RepVGG: Making VGG-style ConvNets great again. In Proceedings of the IEEE Conference on Computer Vision and Pattern Recognition (CVPR). https://doi.org/10.1109/CVPR46437.2021.01352

[13] Zidong Du, Robert Fasthuber, Tianshi Chen, Paolo Lenne, Ling Li, Tao Luo, Xiaobing Feng, Yunji Chen, and Olivier Temam. 2015. ShiDianNao: Shifting vision processing closer to the sensor. In Proceedings of the 42nd ACM/IEEE International Symposium on Computer Architecture (ISCA). https://doi.org/10.1145/2749469.2750389

[14] Mingyu Gao, Jing Pu, Xuan Yang, Mark Horowitz, and Christos Kozyrakis. 2017. TETRIS: Scalable and efficient neural network acceleration with 3D memory. In Proceedings of the 22nd International Conference on Architectural Support for Programming Languages and Operating Systems (ASPLOS). https://doi.org/10.1145/3037697.3037702

[15] Gartner. 2020. Gartner Highlights 10 Uses for AI-Powered Smartphones. https://www.gartner.com/en/newsroom/ press-releases/2018-03-20-gartner-highlights-10-uses-for-ai-powered-smartphones.

[16] Google. 2018. Edge TPU. https://cloud.google.com/edge-tpu.

[17] Google. 2019. Pixel 4 is here to help. https://blog.google/products/pixel/pixel-4/.

[18] Sumanth Gudaparthi, Surya Narayanan, Rajeev Balasubramonian, Edouard Giacomin, Hari Kambalasubramanyam, and Pierre-Emmanuel Gaillardon. 2019. Wire-aware architecture and dataflow for CNN accelerators. In Proceedings of the 50th Annual IEEE/ACM International Symposium on Microarchitecture (MICRO). https://doi.org/10.1145/3352460. 3358316

[19] Suyog Gupta and Berkin Akin. 2020. Accelerator-aware neural network design using AutoML. In Proceeding of the Conference on Machine Learning and Systems (MLSys). https://arxiv.org/abs/2003.02838. 
[20] Mathew Hall and Vaughn Betz. 2020. From TensorFlow graphs to LUTs and wires: Automated sparse and physically aware CNN hardware generation. In Proceeding of the IEEE International Conference on Field-Programmable Technology (ICFPT). https://doi.org/10.1109/ICFPT51103.2020.00017

[21] Kaiming He, Xiangyu Zhang, Shaoqing Ren, and Jian Sun. 2016. Deep residual learning for image recognition. In Proceedings of the IEEE Conference on Computer Vision and Pattern Recognition (CVPR). https://doi.org/10.1109/CVPR. 2016.90

[22] Kaiming He, Xiangyu Zhang, Shaoqing Ren, and Jian Sun. 2016. Identity mappings in deep residual networks. In Proceedings of the IEEE Conference on Computer Vision and Pattern Recognition (ECCV). http://arxiv.org/abs/1603.05027.

[23] Geoffrey Hinton, Oriol Vinyals, and Jeff Dean. 2015. Distilling the knowledge in a neural network. (2015). arXiv:1503. 02531 http://arxiv.org/abs/1503.02531.

[24] Andrew G. Howard, Menglong Zhu, Bo Chen, Dmitry Kalenichenko, Weijun Wang, Tobias Weyand, Marco Andreetto, and Hartwig Adam. 2017. MobileNets: Efficient convolutional neural networks for mobile vision applications. (2017). arXiv:1704.04861 http://arxiv.org/abs/1704.04861.

[25] Jie Hu, Li Shen, Samuel Albanie, Gang Sun, and Enhua Wu. 2018. Squeeze-and-excitation networks. In Proceedings of the IEEE Conference on Computer Vision and Pattern Recognition (CVPR). https://doi.org/10.1109/CVPR.2018.00745

[26] Gao Huang, Zhuang Liu, Laurens Van Der Maaten, and Kilian Q. Weinberger. 2017. Densely connected convolutional networks. In Proceedings of the IEEE Conference on Computer Vision and Pattern Recognition (CVPR). https://doi.org/ 10.1109/CVPR.2017.243

[27] Sergey Ioffe and Christian Szegedy. 2015. Batch normalization: Accelerating deep network training by reducing internal covariate shift. In Proceedings of the International Conference on Machine Learning (ICML). http://proceedings.mlr. press/v37/ioffe15.html.

[28] Norman P. Jouppi, Doe Hyun Yoon, Matthew Ashcraft, Mark Gottscho, Thomas B. Jablin, George Kurian, James Laudon, Sheng Li, Peter Ma, Xiaoyu Ma, Thomas Norrie, Nishant Patil, Sushma Prasad, Cliff Young, Zongwei Zhou, and David Patterson. 2021. Ten lessons from three generations shaped Google's TPUv4i. In Proceedings of the 48th ACM/IEEE International Symposium on Computer Architecture (ISCA). https://doi.org/10.1109/ISCA52012.2021.00010

[29] Norman P. Jouppi, Doe Hyun Yoon, George Kurian, Sheng Li, Nishant Patil, James Laudon, Cliff Young, and David Patterson. 2020. A domain-specific supercomputer for training deep neural networks. Commun. ACM (2020). https: //doi.org/10.1145/3360307

[30] Norman P. Jouppi, Cliff Young, Nishant Patil, David Patterson, Gaurav Agrawal, Raminder Bajwa, Sarah Bates, Suresh Bhatia, Nan Boden, Al Borchers, Rick Boyle, Pierre-Luc Cantin, Clifford Chao, Chris Clark, Jeremy Coriell, Mike Daley, Matt Dau, Jeffrey Dean, Ben Gelb, Tara Vazir Ghaemmaghami, Rajendra Gottipati, William Gulland, Robert Hagmann, C. Richard Ho, Doug Hogberg, John Hu, Robert Hundt, Dan Hurt, Julian Ibarz, Aaron Jaffey, Alek Jaworski, Alexander Kaplan, Harshit Khaitan, Daniel Killebrew, Andy Koch, Naveen Kumar, Steve Lacy, James Laudon, James Law, Diemthu Le, Chris Leary, Zhuyuan Liu, Kyle Lucke, Alan Lundin, Gordon MacKean, Adriana Maggiore, Maire Mahony, Kieran Miller, Rahul Nagarajan, Ravi Narayanaswami, Ray Ni, Kathy Nix, Thomas Norrie, Mark Omernick, Narayana Penukonda, Andy Phelps, Jonathan Ross, Matt Ross, Amir Salek, Emad Samadiani, Chris Severn, Gregory Sizikov, Matthew Snelham, Jed Souter, Dan Steinberg, Andy Swing, Mercedes Tan, Gregory Thorson, Bo Tian, Horia Toma, Erick Tuttle, Vijay Vasudevan, Richard Walter, Walter Wang, Eric Wilcox, and Doe Hyun Yoon. 2017. In-datacenter performance analysis of a tensor processing unit. In Proceedings of the 44th ACM/IEEE International Symposium on Computer Architecture (ISCA). https://doi.org/10.1145/3079856.3080246

[31] Byeongho Kim, Jongwook Chung, Eojin Lee, Wonkyung Jung, Sunjung Lee, Jaewan Choi, Jaehyun Park, Minbok Wi, Sukhan Lee, and Jung Ho Ahn. 2020. MViD: Sparse matrix-vector multiplication in mobile DRAM for accelerating recurrent neural networks. IEEE Trans. Comput. 69 (2020), 955-967. https://doi.org/10.1109/TC.2020.2984496

[32] Hyoukjun Kwon, Prasanth Chatarasi, Michael Pellauer, Angshuman Parashar, Vivek Sarkar, and Tushar Krishna. 2019. Understanding reuse, performance, and hardware cost of DNN dataflows: A data-centric approach. In Proceedings of the 52nd Annual IEEE/ACM International Symposium on Microarchitecture (MICRO). https://doi.org/10.1145/3352460. 3358252

[33] Hyoukjun Kwon, Ananda Samajdar, and Tushar Krishna. 2018. MAERI: Enabling flexible dataflow mapping over DNN accelerators via reconfigurable interconnects. In Proceedings of the 22nd International Conference on Architectural Support for Programming Languages and Operating Systems (ASPLOS). https://doi.org/10.1145/3173162.3173176

[34] Sukhan Lee, Hyunyoon Cho, Young Hoon Son, Yuhwan Ro, Nam Sung Kim, and Jung Ho Ahn. 2018. Leveraging power-performance relationship of energy-efficient modern DRAM devices. IEEE Access 6 (2018), 31387-31398. https: //doi.org/10.1109/ACCESS.2018.2845861

[35] Jiajun Li, Guihai Yan, Wenyan Lu, Shuhao Jiang, Shijun Gong, Jingya Wu, and Xiaowei Li. 2018. SmartShuttle: Optimizing off-chip memory accesses for deep learning accelerators. In Design, Automation and Test in Europe Conference (DATE). https://doi.org/10.23919/DATE.2018.8342033 
[36] Sheng Li, Jung Ho Ahn, Richard D. Strong, Jay B. Brockman, Dean M. Tullsen, and Norman P. Jouppi. 2009. McPAT: An integrated power, area, and timing modeling framework for multicore and manycore architectures. In Proceedings of the 42nd Annual IEEE/ACM International Symposium on Microarchitecture (MICRO). https://doi.org/10.1145/1669112. 1669172

[37] Heng Liao, Jiajin Tu, Jing Xia, and Xiping Zhou. 2019. A scalable unified architecture for neural network computing from nano-level to high performance computing. In IEEE Hot Chips Symposium (HCS). https://doi.org/10.1109/ HOTCHIPS.2019.8875654

[38] NVIDIA. 2018. The NVIDIA deep learning accelerator. In IEEE Hot Chips Symposium (HCS).

[39] Angshuman Parashar, Priyanka Raina, Yakun Sophia Shao, Yu-Hsin Chen, Victor A. Ying, Anurag Mukkara, Rangharajan Venkatesan, Brucek Khailany, Stephen W. Keckler, and Joel Emer. 2019. Timeloop: A systematic approach to DNN accelerator evaluation. In Proceedings of the IEEE International Symposium on Performance Analysis of Systems and Software (ISPASS). https://doi.org/10.1109/ISPASS.2019.00042

[40] Hieu Pham, Zihang Dai, Qizhe Xie, Minh-Thang Luong, and Quoc V. Le. 2020. Meta pseudo labels. (2020). arXiv:2003. 10580 https://arxiv.org/abs/2003.10580.

[41] Esteban Real, Alok Aggarwal, Yanping Huang, and Quoc V. Le. 2019. Regularized evolution for image classifier architecture search. In Proceedings of the Conference on Association for the Advancement of Artificial Intelligence (AAAI). https://doi.org/10.1609/aaai.v33i01.33014780

[42] Jonathan Ross and Andrew Everett Pheps. 2015. Computing Convolutions Using a Neural Network Processor. US Patent App. 62/164,902.

[43] Jonathan Ross and Gregory Michael Thorson. 2015. Rotating Data for Neural Network Computations. US Patent App. 62/164,908.

[44] Mark Sandler, Andrew Howard, Menglong Zhu, Andrey Zhmoginov, and Liang-Chieh Chen. 2018. MobileNetV2: Inverted residuals and linear bottlenecks. In Proceedings of the IEEE Conference on Computer Vision and Pattern Recognition (CVPR). https://doi.org/10.1109/CVPR.2018.00474

[45] Karen Simonyan and Andrew Zisserman. 2015. Very deep convolutional networks for large-scale image recognition. In Proceedings of the International Conference on Learning Representations (ICLR). http://arxiv.org/abs/1409.1556.

[46] Jinook Song, Yunkyo Cho, Jun-Seok Park, Jun-Woo Jang, Sehwan Lee, Joon-Ho Song, Jae-Gon Lee, and Inyup Kang. 2019. An 11.5TOPS/W 1024-MAC butterfly structure dual-core sparsity-aware neural processing unit in $8 \mathrm{~nm}$ flagship mobile SoC. In Proceedings of the IEEE International Solid-State Circuits Conference (ISSCC). https://doi.org/10.1109/ ISSCC.2019.8662476

[47] Statista. 2020. Forecast number of mobile users worldwide from 2020 to 2024. https://www.statista.com/statistics/ 218984/number-of-global-mobile-users-since-2010/.

[48] Chen Sun, Abhinav Shrivastava, Saurabh Singh, and Abhinav Gupta. 2017. Revisiting unreasonable effectiveness of data in deep learning era. In Proceedings of the IEEE International Conference on Computer Vision (ICCV). https://doi. org/10.1109/ICCV.2017.97

[49] Christian Szegedy, Wei Liu, Yangqing Jia, Pierre Sermanet, Scott Reed, Dragomir Anguelov, Dumitru Erhan, Vincent Vanhoucke, and Andrew Rabinovich. 2015. Going deeper with convolutions. In Proceedings of the IEEE Conference on Computer Vision and Pattern Recognition (CVPR). https://doi.org/10.1109/CVPR.2015.7298594

[50] Christian Szegedy, Vincent Vanhoucke, Sergey Ioffe, Jon Shlens, and Zbigniew Wojna. 2016. Rethinking the inception architecture for computer vision. In Proceedings of the IEEE Conference on Computer Vision and Pattern Recognition (CVPR). https://doi.org/10.1109/CVPR.2016.308

[51] Mingxing Tan, Bo Chen, Ruoming Pang, Vijay Vasudevan, Mark Sandler, Andrew Howard, and Quoc V. Le. 2019. MnasNet: Platform-aware neural architecture search for mobile. In Proceedings of the IEEE Conference on Computer Vision and Pattern Recognition (CVPR). https://doi.org/10.1109/CVPR.2019.00293

[52] Mingxing Tan and Quoc V. Le. 2019. EfficientNet: Rethinking model scaling for convolutional neural networks. (2019). arXiv:1905.11946 http://arxiv.org/abs/1905.11946.

[53] Shyamkumar Thoziyoor, Jung Ho Ahn, Matteo Monchiero, Jay B. Brockman, and Norman P. Jouppi. 2008. A comprehensive memory modeling tool and its application to the design and analysis of future memory hierarchies. In Proceedings of the 35th ACM/IEEE International Symposium on Computer Architecture (ISCA). https://doi.org/10.1109/ ISCA.2008.16

[54] Hugo Touvron, Andrea Vedaldi, Matthijs Douze, and Hervé Jégou. 2020. Fixing the train-test resolution discrepancy: FixEfficientNet. (2020). arXiv:2003.08237 https://arxiv.org/abs/2003.08237.

[55] Rangharajan Venkatesan, Yakun Sophia Shao, Brian Zimmer, Jason Clemons, Matthew Fojtik, Nan Jiang, Ben Keller, Alicia Klinefelter, Nathaniel Pinckney, Priyanka Raina, Stephen G. Tell, Yanqing Zhang, William J. Dally, Joel S. Emer, C. Thomas Gray, Stephen W. Keckler, and Brucek Khailany. 2019. A 0.11 PJ/OP, 0.32-128 tops, scalable multi-chipmodule-based deep neural network accelerator designed with a high-productivity VLSI methodology. In IEEE Hot Chips Symposium (HCS). https://doi.org/10.1109/HOTCHIPS.2019.8875657 
[56] Ofri Wechsler, Michael Behar, and Bharat Daga. 2019. Spring Hill (NNP-I 1000) Intel's data center inference chip. In IEEE Hot Chips Symposium (HCS). https://doi.org/10.1109/HOTCHIPS.2019.8875671

[57] Hao-Ning Wu and Chih-Tsun Huang. 2019. Data locality optimization of depthwise separable convolutions for CNN inference accelerators. In Design, Automation and Test in Europe Conference and Exhibition (DATE). https://doi.org/10. 23919/DATE.2019.8715097

[58] Qizhe Xie, Minh-Thang Luong, Eduard Hovy, and Quoc V. Le. 2020. Self-training with noisy student improves ImageNet classification. In Proceedings of the IEEE Conference on Computer Vision and Pattern Recognition (CVPR). https://doi.org/10.1109/CVPR42600.2020.01070

[59] Saining Xie, Ross Girshick, Piotr Dollár, Zhuowen Tu, and Kaiming He. 2017. Aggregated residual transformations for deep neural networks. In Proceedings of the IEEE Conference on Computer Vision and Pattern Recognition (CVPR). https://doi.org/10.1109/CVPR.2017.634

[60] Rui Xu, Sheng Ma, Yaohua Wang, and Yang Guo. 2020. CMSA: Configurable multi-directional systolic array for convolutional neural networks. In Proceedings of the IEEE Conference on Computer Design (ICCD). https://doi.org/10.1109/ ICCD50377.2020.00089

[61] Andrew Yang. 2019. Deep learning training at Scale Spring Crest deep learning accelerator. In IEEE Hot Chips Symposium (HCS). https://doi.org/10.1109/HOTCHIPS.2019.8875643

[62] Yunxuan Yu, Tiandong Zhao, Kun Wang, and Lei He. 2020. Light-OPU: An FPGA-based overlay processor for lightweight convolutional neural networks. In Proceedings of the ACM/SIGDA International Symposium on FieldProgrammable Gate Arrays (FPGA). https://doi.org/10.1145/3373087.3375311

[63] Barret Zoph, Vijay Vasudevan, Jonathon Shlens, and Quoc V. Le. 2018. Learning transferable architectures for scalable image recognition. In Proceedings of the IEEE Conference on Computer Vision and Pattern Recognition (CVPR). https: //doi.org/10.1109/CVPR.2018.00907

Received June 2021; revised September 2021; accepted November 2021 